\title{
On the Graphs of Hoffman-Singleton and Higman-Sims
}

\author{
Paul R. Hafner \\ Department of Mathematics \\ University of Auckland \\ Auckland (New Zealand) \\ hafner@math. auckland.ac.nz
}

Submitted: Mar 2, 2004; Accepted: Aug 27, 2004; Published: Nov 3, 2004

MR Subject Classifications: 05C62, 05C25; 05B25, 51E10, 51E26

Keywords: Hoffman-Singleton graph, Higman-Sims graph, Higman-Sims group, biaffine plane,

$\mathrm{S}(3,6,22)$

\begin{abstract}
We propose a new elementary definition of the Higman-Sims graph in which the 100 vertices are parametrised with $\mathbb{Z}_{4} \times \mathbb{Z}_{5} \times \mathbb{Z}_{5}$ and adjacencies are described by linear and quadratic equations. This definition extends Robertson's pentagonpentagram definition of the Hoffman-Singleton graph and is obtained by studying maximum cocliques of the Hoffman-Singleton graph in Robertson's parametrisation. The new description is used to count the 704 Hoffman-Singleton subgraphs in the Higman-Sims graph, and to describe the two orbits of the simple group HS on them, including a description of the doubly transitive action of HS within the HigmanSims graph. Numerous geometric connections are pointed out. As a by-product we also have a new construction of the Steiner system $S(3,6,22)$.
\end{abstract}

\section{Introduction}

The Higman-Sims graph is the unique strongly regular graph whose parameters are $(100,22,0,6)$, i.e. it is a graph of order 100 , regular of degree 22 ; it is triangle-free (any two adjacent vertices have 0 common neighbours), and any two non-adjacent vertices have exactly 6 neighbours in common. This graph made its first official appearance [23] in the context of the construction of the sporadic simple group HS which is a subgroup of index 2 in the automorphism group of the graph (note Section 13 for a comment on the history).

In this paper we provide a new and elementary construction of the Higman-Sims graph, combining a geometric interpretation [16] of Robertson's pentagon-pentagram construction of the Hoffman-Singleton graph with the known construction of the Higman-Sims 
graph via maximum cocliques in the Hoffman-Singleton graph. We demonstrate the flavour of the construction by exploring some automorphisms, counting the HoffmanSingleton subgraphs and describing the doubly transitive action of degree 176 of the sporadic simple group HS from within the Higman-Sims graph. These applications build also on the combinatorial studies of the Hoffman-Singleton graph by James [30]. The key result of [30] is that any 5-cycle in the Hoffman-Singleton graph determines a split of the graph into two sets of five 5-cycles which are related by Robertson's pentagon-pentagram equations. It will become apparent that this extends to the Higman-Sims graph: every 5-cycle determines a split into four sets of five 5-cycles.

Structure of the paper. We give some background information about the HoffmanSingleton graph in Section 2. Section 3 contains our new construction of the Higman-Sims graph. A first verification that the given graph is the Higman-Sims graph is given as Theorem 1 whose proof is left as an exercise. Section 4 introduces some of the automorphisms of the graph which can be used to show that the Higman-Sims graph is in fact a Cayley graph. These automorphisms also give a hint of the remarkable symmetries of this graph. Sections 5 and 6 show how to derive the new definition from the description of the Higman-Sims graph as (modified) incidence graph of the vertices of the HoffmanSingleton graph and one family of its maximum cocliques. This is achieved by extending the parametrisation of the Hoffman-Singleton graph in Definition 1 to a parametrisation of the maximum cocliques, allowing adjacencies (incidences and certain intersection properties) to be expressed in the form of simple equations (Fig. 2) without any reference to cocliques. Along the way we highlight some properties of maximum cocliques in the Hoffman-Singleton graph. The well-known existence of two families of maximum cocliques (containing 50 cocliques each) is captured very effectively by our parametrisation. Section 7 extends our definition of the Higman-Sims graph to a graph of order 150 which encapsulates everything about maximum cocliques of the Hoffman-Singleton graph. In Section 8 we show how to count the Hoffman-Singleton subgraphs in the Higman-Sims graph and characterise their two orbits under HS by means of certain intersection numbers. Section 9 is a brief sidetrack to demonstrate that some classical gems are explicitly present in the Higman-Sims graph: from the correspondence between lines of PG $(3,2)$ and triples of a 7-element set to (almost) the exceptional isomorphism between the alternating group $A_{8}$ and $P S L(4,2)$, as well as the Alt(7) and Alt(8) geometries. In Section 10 we demonstrate the doubly transitive action of HS on 176 points as it manifests itself within the Higman-Sims graph. Section 11 picks up the geometric theme again, showing that the adjacencies of the Higman-Sims graph can be understood in terms of geometric relationships between points, lines, conics and dual conics in a biaffine plane, with strong connections to Wild's semibiplanes [49]. In Section 12 we highlight a decomposition of the Higman-Sims graph into 5 isomorphic subgraphs of order 20, concluding with a brief historical note in Section 13.

In the remainder of this introduction, we give a brief overview of some constructions of the Higman-Sims graph, and establish the notational conventions for the rest of the paper.

Constructions of the Higman-Sims graph. The original construction by Higman 
and Sims [23] is based on the Steiner system $S(3,6,22)$. This construction is visible in Fig. 4, if one considers only $\mathrm{H}_{3} \cup \mathrm{H}_{2}$. In a variation on this theme, [2] begins with the projective plane of order 4 and effectively incorporates some of the construction steps of $S(3,6,22)$ into the construction of the Higman-Sims graph. Elsewhere [18], we will describe the extension of $S(2,5,21)$ to $S(5,8,24)$ from within the Higman-Sims graph.

It is known that the maximum cocliques of the Hoffman-Singleton graph form a graph with two connected components (each isomorphic to the Hoffman-Singleton graph) if adjacency is defined by disjointness. This allows to construct the Higman-Sims graph either by introducing additional edges between those cocliques which meet in 8 vertices, or else one can take the original Hoffman-Singleton graph together with one of the connected components of the max-coclique graph, defining further adjacencies by incidence. This latter approach is the basis of our new construction. A neat unification of these methods leads to a graph of order 150 ([5], p.108, [6], p.394, cf. Section 7 below).

In [35] Mathon and Street present 'the first elementary construction of the HigmanSims graph, starting from scratch without having to refer to cocliques in the HoffmanSingleton graph.' Their interesting construction should be seen as describing an occurrence of the Higman-Sims graph in an unexpected place, perhaps stretching the meaning of the word 'elementary'. Another elementary description of the Higman-Sims graph is its representation as a Cayley graph, found independently by Heinze [21], Jørgensen-Klin [32] and Praeger-Schneider [44] (cf. Theorem 3).

Apart from the Cayley graph construction, there are other group-theoretic approaches to the Higman-Sims graph, for example [10]. In Remark 28 we will indicate a construction based on an incidence graph combined with a group action.

Hughes [27] uses semisymmetric 3-designs, while Yoshiara [52] has a construction of the Higman-Sims graph with vertices in the Leech lattice. A comprehensive description of the Higman-Sims graph (and G. Higman's related geometry) in the Leech lattice appears in R.A. Wilson's paper [51].

Notation and Terminology. The following notation will be used throughout this paper:

$\mathbb{Z}_{5}$ denotes the field of order $5, \mathbb{Z}_{5}^{*}$ its multiplicative group.

$G$ will always denote the graph defined in Definition 2 (which is the Higman-Sims graph, cf. Theorem 1 and Remark 6).

$V_{i}(i=0, \ldots, 5)$ are sets of 25 elements $(i, x, y), x, y \in \mathbb{Z}_{5}$; elements $(0, x, y) \in V_{0}$ will sometimes be referred to as point vertices, and in Section 5 just as points $(x, y)$. Similarly for line vertices $(1, m, c) \in V_{1}$; these are also referred to as "the line $y=m x+c$ " in Section 5.

$H, H_{1}, H_{2}, H_{3}$ denote Hoffman-Singleton graphs: $H$ and $H_{1}$ will have $V_{0} \cup V_{1}$ as vertex set; the vertex set of $H_{2}$ is $V_{2} \cup V_{3}$ and for $H_{3}$ it is $V_{4} \cup V_{5}$.

$K$ denotes the supergraph of order 150, defined in Section 7; vertex set: $V_{0} \cup \cdots \cup V_{5}$. $\operatorname{Aut}(X)$ denotes the automorphism group of a graph $X$.

HS denotes the index 2 subgroup of $\operatorname{Aut}(G)$, consisting of all even permutations of the vertices of $G$ (cf. Remark 14 and Section 10); this is the Higman-Sims group. $g, h$ are special automorphisms of the Higman-Sims graph, defined in Lemma 12. 
In [16] we introduced the term affine automorphism to denote automorphisms of $H$ which preserve the partition $\left\{V_{0}, V_{1}\right\}$ (they are induced by collineations or correlations of the biaffine plane).

A set of five disjoint 5-cycles with no further edges between any vertices will be denoted by $5 C_{5}$.

Numbering of items: there are three distinct numbering schemes: Remarks and Lemmas are in one sequence; Definitions and Theorems each have a sequence of their own.

Web resources for this paper: some MaGma [3] files and links related to this paper are available at [15].

\section{The Hoffman-Singleton Graph}

The Hoffman-Singleton graph is the unique Moore graph of degree $7[26,6]$. There are essentially three constructions of this graph which may be described succinctly as " $1+7+42 "$ " " $15+35 "$, and " $25+25 "$. For our purposes, Robertson's [45] pentagonpentagram construction ( $25+25$ ") with the geometric interpretation in the affine plane $\mathrm{AG}(2,5)$ from [16] is pivotal and given as Definition 1 below. The " $15+35$ " construction is related to the projective space $\mathrm{PG}(3,2)$ and will come into focus in Section 8 and Remark 45, whilst the Moore graph definition (" $1+7+42$ ") is visible in Fig. $4\left(H_{3}\right)$.

Definition 1. The Hoffman-Singleton graph $H$ has vertex set $\mathbb{Z}_{2} \times \mathbb{Z}_{5} \times \mathbb{Z}_{5}$ and the following edges:

$$
\begin{array}{rllll}
(0, x, y) & \text { is adjacent to } & \left(0, x, y^{\prime}\right) & \text { if and only if } & y-y^{\prime}= \pm 1 \\
(1, m, c) & \text { is adjacent to } & \left(1, m, c^{\prime}\right) & \text { if and only if } & c-c^{\prime}= \pm 2 \\
(0, x, y) & \text { is adjacent to } & (1, m, c) & \text { if and only if } & y=m x+c .
\end{array}
$$

In [16] we showed that the geometry of the pentagon-pentagram construction does not lie in the pentagons and pentagrams but in the adjacency rules $y=m x+c$. Under this geometric point of view the Hoffman-Singleton graph is the incidence graph of a biaffine plane with pentagons and pentagrams as additional edges. (A biaffine plane is an affine plane with one parallel class of lines - the 'vertical' lines, in our coordinatisation omitted. These structures inherit the best features of both projective and affine geometry: duality and parallelism.) In this spirit, we refer to vertices $(0, x, y)$ as points and to vertices $(1, m, c)$ as lines. Fig. 1 summarises Definition 1, introducing also the notation $V_{0}=\left\{(0, x, y): x, y \in \mathbb{Z}_{5}\right\}, V_{1}=\left\{(1, m, c): m, c \in \mathbb{Z}_{5}\right\}$.

We recall from [16] that two parallel lines $(1, m, c)$ and $\left(1, m, c^{\prime}\right)$ of $H$ are adjacent if and only if their points of intersection $(0, x, y)$ and $\left(0, x, y^{\prime}\right)$ with any vertical line are non-adjacent. A particular consequence of this is the existence of 1255 -cycles in $H$ which consist of two adjacent points on a vertical line and three consecutive lines, e.g. $(0,0,0),(0,0,1),(1,0,1),(1,0,3),(1,0,0)$ (and the same with 3 points and 2 lines). Each of these 5 -cycles determines a distinct split of $H$ into a pair of $5 C_{5}$ (cf. [30] or [16]) which 

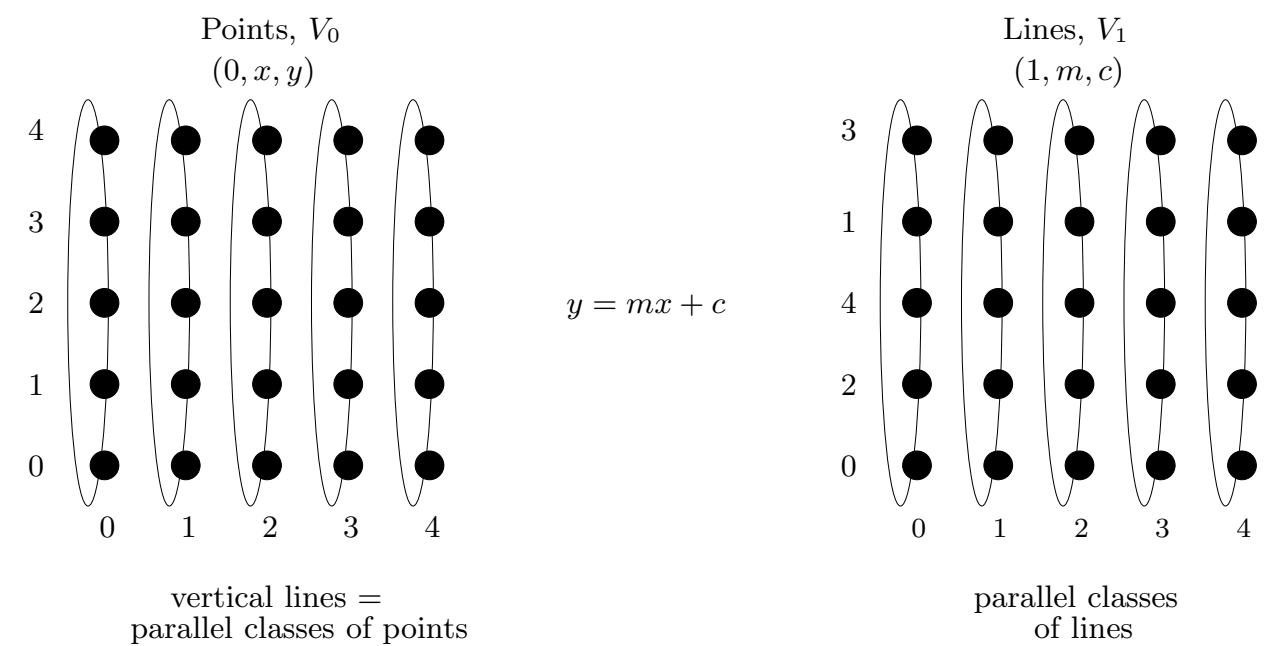

Figure 1: Geometric interpretation of Robertson's description of the Hoffman-Singleton graph

can be labelled as in Fig. 1 with the same adjacency rules. The 2 -fold transitivity of the automorphism group of the Hoffman-Singleton graph on the 126 splits now follows from the transitivity on these special 5 -cycles of the group of affine automorphisms (which stabilises the obvious split into the given pair of $5 C_{5}$ ).

Remark 1. We note at this point that the enumeration of 5-cycles in [16] shows that any 5 -cycle of $H$ has $0,2,3$, or 5 vertices in common with $V_{0}$. Now assume that $\left\{V_{0}^{\prime}, V_{1}^{\prime}\right\} \neq$ $\left\{V_{0}, V_{1}\right\}$ is a split of $H$ into a pair of complementary $5 C_{5}$ and that $V_{0}^{\prime}$ contains one of the above-mentioned special 5 -cycles with exactly two consecutive vertices $u, v$ on a vertical line, with the remaining three consecutive vertices $r, s, t$ all in a fixed parallel class of lines (in $V_{1}$ ). Then $r, s, t$ have distinct Neighbours on each of the 5-cycles in $V_{0}$, and therefore no vertical line can contribute more than 2 vertices to $V_{0}^{\prime}$. Therefore $\left|V_{0}^{\prime} \cap V_{0}\right|=10$ in this case. Dually, if $V_{0}^{\prime}$ contains a 5 -cycle with 3 consecutive points on a vertical line then $\left|V_{0}^{\prime} \cap V_{0}\right|=15$. This will be useful in the proof of Lemma 34 .

The biaffine plane underlying our description of the Hoffman-Singleton graph inherits a duality from the projective geometry into which it can be embedded. An example of such a mapping $\psi$ which interchanges points and lines and preserves all adjacencies is

$$
(0, x, y) \stackrel{\psi}{\mapsto}(1, x, 2 y), \quad(1, m, c) \stackrel{\psi}{\mapsto}(0,3 m, 2 c) .
$$

Whenever we need to interchange points and lines, we might use a phrase like 'by duality'.

Remark 2. This paper as well as its precursor [16] can be seen under the following general viewpoint. When the Petersen graph is viewed as a pair of 5-cycles, one immediately sees 20 of its automorphisms (dihedral group for the cycle, and swapping the cycles). The full automorphism group, however, has order 120, due to the fact that there are 6 distinct ways 
of choosing a pair of 'opposite' 5-cycles. The same holds for the Hoffman-Singleton graph: looking at the split into points and lines of a biaffine plane, one immediately sees 2000 affine automorphisms; the full automorphism group, however, has order 252000 because there are 126 distinct splits into points and lines of a biaffine plane. We will note the same for the Higman-Sims graph later in this paper: when we consider the Higman-Sims graph as a pair of Hoffman-Singleton graphs, we can immediately see 252000 automorphisms. But the total number of automorphisms is $352 \cdot 252000$, since there are 352 ways of splitting the Higman-Sims graph into a pair of Hoffman-Singleton graphs. The same phenomenon was observed [19] on a graph of order 32, the smallest of the McKay-Miller-Šríńn graphs for $q=2$.

The remainder of this section deals with non-affine automorphisms of the HoffmanSingleton graph, showing how they arise from automorphisms of the Petersen graph. It is obvious that any of the 5 -cycles of $V_{0}$ together with any of the 5 -cycles of $V_{1}$ induce a Petersen graph in $H$. When considering automorphisms of $H$, we might therefore look at extending automorphisms of a Petersen graph.

Lemma 3. Let $P$ be a Petersen subgraph of $H$. Then every automorphism of $P$ can be extended to an automorphism of $H$ in exactly four ways.

Proof. Implicit in the uniqueness proof [30] of the Hoffman-Singleton graph $H$ is a proof that $\operatorname{Aut}(H)$ is transitive on the 525 Petersen subgraphs of $H$ and that we may assume the vertices of $P$ to be $(0,0,0), \ldots,(0,0,4),(1,0,0), \ldots,(1,0,4)$. Then it follows from the orbit-stabiliser theorem that the stabiliser of $P$ in $\operatorname{Aut}(H)$ has order $252000 / 525=480$. The identity of $P$ has 4 extensions to an automorphism of $H$, since we are free to choose an eigenvalue in the horizontal direction (4 possibilities). Therefore the stabiliser of $P$ induces 120 distinct automorphism of $P$, i.e. every automorphism of $P$ can be extended to an automorphism of $H$.

Remark 4. We give an example of a (non-affine) automorphism of $P$, and an extension to $H$, since this will be useful later on. It is easy enough to construct an automorphism of $P$ : just choose any 5-cycle, and find its complementary cycle. We indicate this by listing the images of the vertices of $P$ in a scheme according to Fig. 1. We also list the image of the additional vertex $(0,1,3)$. This image was determined as follows: the unique neighbour of $(0,1,3)$ in $P$ is $(1,0,3)$ which is mapped to $(1,0,4)$ under the automorphism of $P$. Therefore the image of $(0,1,3)$ must be one of the 4 neighbours of $(1,0,4)$ outside $P$; our choice was $(0,4,4)$. For better orientation we have labelled the rows as they are labelled in Fig. 1, cycles in the left hand block $V_{0}$ being labelled differently from those in the right hand block $V_{1}$.

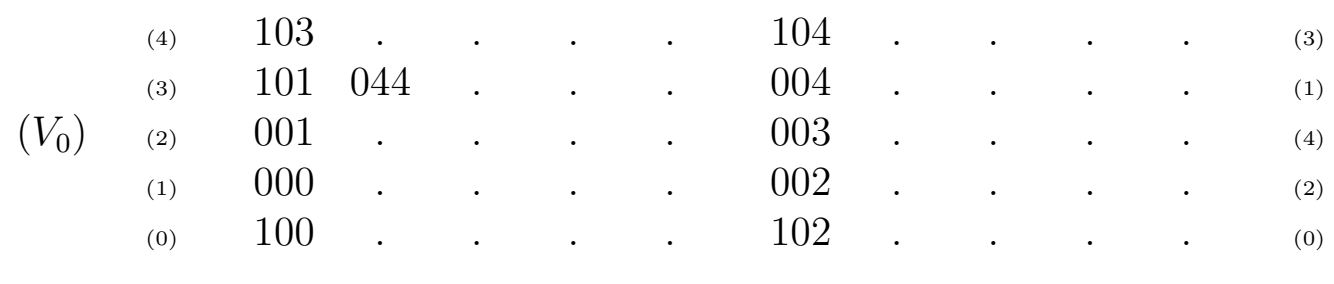


The construction of the automorphism of $H$ is now mechanical (and best left to a computer, although it is easy enough to do it by hand). The key ingredient is that $H$ is an $\operatorname{srg}(50,7,0, \mathbf{1})$, and that if one starts with a subgraph $X$ of $H$ which contains $P$ and at least one more vertex, one obtains all of $H$ by successively adding common neighbours of pairs of non-adjacent vertices. (The Petersen graph, being an $\operatorname{srg}(10,3,0,1)$, is closed under the operation of taking 'midpoints' of non-adjacent vertices.) For example, to determine the image of $v=(1,3,0)$, note that $v$ is the unique common neighbour of $(0,0,0)$ and $(0,1,3)$, both of whose images are already known: $(1,0,0)$ and $(0,4,4)$. The image of $v$ must therefore be the unique common neighbour of these two vertices, i.e. $(0,4,0)$. After a bit of work one obtains the following automorphism of the graph $H$. The significance of the boldface entries will be explained in Section 10 .

$\begin{array}{lllllllllllll} & (4) & \mathbf{1 0 3} & \mathbf{1 4 3} & 123 & 133 & \mathbf{1 1 3} & \mathbf{1 0 4} & 021 & \mathbf{0 1 1} & \mathbf{0 4 1} & 031 & { }^{(3)} \\ \left(V_{0}\right) & { }^{(3)} & 101 & 044 & 034 & 024 & 014 & \mathbf{0 0 4} & \mathbf{1 3 0} & 110 & 140 & \mathbf{1 2 0} & { }_{(1)} \\ & (2) & 001 & \mathbf{1 3 2} & \mathbf{1 4 2} & \mathbf{1 1 2} & \mathbf{1 2 2} & 003 & 013 & 033 & 023 & 043 & { }_{(4)} \\ & (1) & 000 & 134 & 144 & 114 & 124 & 002 & \mathbf{1 2 1} & \mathbf{1 4 1} & \mathbf{1 1 1} & \mathbf{1 3 1} & { }_{(2)} \\ & (0) & \mathbf{1 0 0} & 012 & \mathbf{0 2 2} & \mathbf{0 3 2} & 042 & 102 & 020 & 010 & 040 & 030 & (0)\end{array}$

\section{A New Definition of the Higman-Sims Graph}

Definition 2. Throughout this paper, $G$ is the graph with vertex set $\mathbb{Z}_{4} \times \mathbb{Z}_{5} \times \mathbb{Z}_{5}$ and adjacencies defined as follows (cf. Figure 2):

$\begin{array}{rrrll}(0, x, y) & \text { is adjacent to } & \left(0, x, y^{\prime}\right) & \Leftrightarrow & y-y^{\prime}= \pm 1 ; \\ (1, m, c) & \text { is adjacent to } & \left(1, m, c^{\prime}\right) & \Leftrightarrow & c-c^{\prime}= \pm 2 ; \\ (2, A, B) & \text { is adjacent to } & \left(2, A, B^{\prime}\right) & \Leftrightarrow & B-B^{\prime}= \pm 1 ; \\ (3, a, b) \text { is adjacent to } & \left(3, a, b^{\prime}\right) & \Leftrightarrow & b-b^{\prime}= \pm 2 ; \\ (0, x, y) & \text { is adjacent to } & (1, m, c) & \Leftrightarrow & y=m x+c ; \\ (1, m, c) & \text { is adjacent to } & (2, A, B) & \Leftrightarrow & c=2(m-A)^{2}+B ; \\ (2, A, B) & \text { is adjacent to } & (3, a, b) & \Leftrightarrow & B=2 A^{2}+3 a A-a^{2}+b ; \\ (3, a, b) & \text { is adjacent to } & (0, x, y) & \Leftrightarrow & y=(x-a)^{2}+b ; \\ (0, x, y) & \text { is adjacent to } & (2, A, B) & \Leftrightarrow & y=3 x^{2}+A x+B \pm 1 ; \\ (1, m, c) & \text { is adjacent to } & (3, a, b) & \Leftrightarrow & c=m^{2}-a m+b \pm 2 .\end{array}$

We further define

$$
V_{i}=\{i\} \times \mathbb{Z}_{5} \times \mathbb{Z}_{5} \quad(i=0, \ldots, 3) .
$$

Remark 5. The definition is summarised in Fig. 2; each of the four sets $V_{0}, \ldots, V_{3}$ consists of five 5 -cycles. They are indicated in the corners of the square, with labels ' $( \pm 1)$ ' 


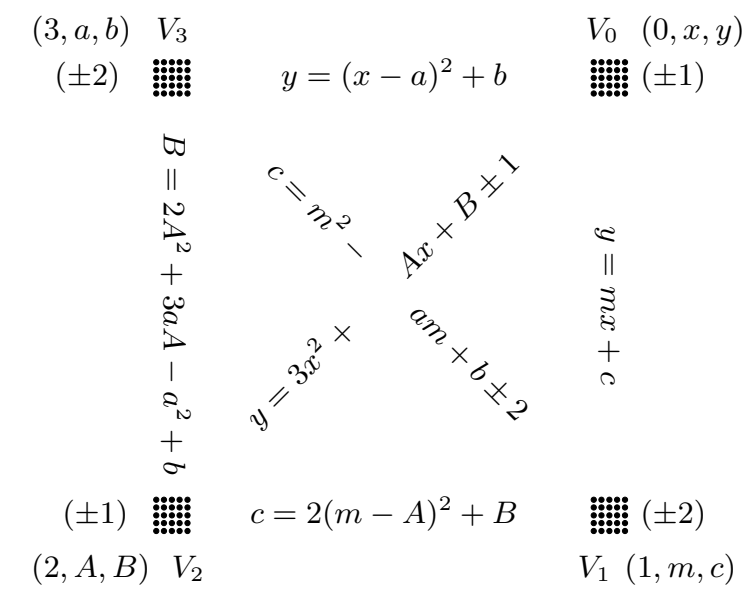

Figure 2: Higman-Sims 'à la Robertson'

to indicate pentagon 5-cycles, and labels ' $( \pm 2)$ ' to indicate pentagram 5-cycles (cf. equations (1), (2), (7)-(10)). Equations between the four sets contain the rules of adjacency. The sets $V_{0}$ and $V_{1}$ together induce a Hoffman-Singleton graph as described in Definition 1. This subgraph is denoted by $H_{1}$ throughout the paper.

Theorem 1. The graph $G$ is strongly regular with parameters $(100,22,0,6)$. This implies that $G$ is the Higman-Sims graph, by the uniqueness theorem of Gewirtz [12].

Remark 6. The proof of Theorem 1 is an exercise in solving quadratic equations over $\mathbb{Z}_{5}$ and can be tackled head-on. We leave the details to the reader. In Section 5 we will take a more gentle approach which indicates how the description given above is obtained, relating it to maximum cocliques in the Hoffman-Singleton graph. This shows that $G$ is the Higman-Sims graph, without having to rely on the characterisation by Gewirtz. Alternatively, one can avoid the use of the theorem of Gewirtz by establishing that given a vertex $x$ of $G$, the edges between vertices at distance 1 and 2 from $x$ form the incidence graph of a $S(3,6,22)$; as shown in [1], p. 273, this can be achieved by an ingenious application of a result by Majindar [34] on block intersections. We note that our construction of the Higman-Sims graph provides also a new construction of $S(3,6,22)$.

As a further alternative, Corollary 22 proves that $G$ is the Higman-Sims graph based on its construction from maximum cocliques in the Hoffman-Singleton graph. The construction from $S(3,6,22)$ is visible in Fig. $4, H_{1} \cup H_{3}$.

It should be pointed out that the proof of Theorem 1 becomes simpler if one makes use of Remark 8 below, as well as taking advantage of the automorphisms which we describe in Section 4. To show that $G$ is triangle-free, one invokes the fact that the Hoffman-Singleton graph is triangle-free and proves by a simple calculation that there do not exist 3 vertices $v_{0}, v_{1}, v_{2}$ with $v_{i} \in V_{i}$ which form a triangle, nor do there exist any $v_{0}, v_{1} \in V_{0}, v_{2} \in V_{2}$ forming a triangle. Similarly, when proving that non-adjacent vertices $u, v$ have 6 common neighbours, only the following cases need to be considered: (1) $v, w \in V_{0}$, belonging to 
the same 5-cycle of $V_{0} ;(2) v, w \in V_{0}$, belonging to distinct 5-cycles of $V_{0} ;(3) v \in V_{0}$, $w \in V_{1} ;(4) v \in V_{0}, w \in V_{2}$.

For a different angle on this, we refer to Remark 25 and Lemma 26.

Remark 7. Alerted by the geometric interpretation of Definition 1, the attentive reader will have noted that for $(i, j) \in\{(0,2),(0,3),(1,2),(1,3)\}$ adjacencies between $V_{i}$ and $V_{j}$ correspond to incidences of certain points or lines with certain parabolas or dual parabolas. Less obvious is that adjacencies between $V_{2}$ and $V_{3}$, as well as those within $V_{2}$ and $V_{3}$, indicate disjointness of certain sets (cf. Corollary 22). Geometric interpretations of all adjacencies between $V_{i}$ and $V_{j}(i \neq j)$ are found in Theorem 6 .

Remark 8. Any two consecutive sets $V_{i}$ and $V_{i+1}$ (where subscripts are taken modulo 4) induce a subgraph of $G$ which is isomorphic to the Hoffman-Singleton graph. We demonstrate this for $i=2$ : the two sets $V_{2}$ and $V_{3}$ each induce five 5 -cycles, the first one arranged as pentagons, the second one arranged as pentagrams, as in the case of $V_{0}$ and $V_{1}$. The vertices $(2, A, B)$ and $(3, a, b)$ are adjacent if and only if $Y=M A+C$ where $Y=B-2 A^{2}$, $C=b-a^{2}, M=3 a$. Thus, after choosing the 0-point on each 5-cycle appropriately (additive adjustments), and after permuting the 5 -cycles in $V_{3}$ (multiplication by 3 ), we get the equations which define the Hoffman-Singleton graph in Definition 1.

Remark 9. The 'diagonal' subgraphs of order 50 induced in $G$ by $V_{0} \cup V_{2}$ and by $V_{1} \cup V_{3}$ have automorphism groups of order 2000, isomorphic to the group of the affine transformations of the Hoffman-Singleton graph (cf. [16]). See Section 11 for more.

\section{Some Automorphisms of $G$}

Automorphisms $\phi$ of $H$ which map $V_{0}$ to itself are mappings $(0, x, y) \mapsto\left(0, x^{\prime}, y^{\prime}\right)$ where $(x, y) \mapsto\left(x^{\prime}, y^{\prime}\right)$ is an affine transformation whose linear part has $(0,1)$ as eigenvector with eigenvalue \pm 1 :

$$
(x, y) \stackrel{\phi}{\mapsto}(x, y)\left(\begin{array}{ll}
r & s \\
0 & t
\end{array}\right)+(e, f)=(r x+e, s x+t y+f),
$$

where $r, t \in \mathbb{Z}_{5}^{*}, s, e, f \in \mathbb{Z}_{5}, t= \pm 1$. Such transformations can be extended readily to automorphisms of the Hoffman-Singleton graph $H_{1}$ (cf. [16]). If we stipulate further that $r^{2}=t$, the mapping $\phi$ can be extended to an automorphism of $G$, preserving each of the sets $V_{i}(i=0, \ldots, 3)$. Note that $(0, x, y) \mapsto(0, x,-y)$ can be extended to an automorphism of $H_{1}$, but not to an automorphism of $G$.

Theorem 2. Let $r, s, t, e, f \in \mathbb{Z}_{5}, t= \pm 1$ and $r^{2}=t$. The mapping $\phi: V_{0} \rightarrow V_{0}$ defined by

$$
(0, x, y)^{\phi}=(0, r x+e, s x+t y+f)
$$


can be extended to an automorphism $\phi$ of $G$ by defining:

$$
\begin{aligned}
(1, m, c)^{\phi} & =(1, r m+r s t,-r m e+t c+f-r e s t) \\
(2, A, B)^{\phi} & =\left(2, r A-e+r s t,-r A e+t B+f-r e s t-2 e^{2}\right), \\
(3, a, b)^{\phi} & =\left(3, r a+e+2 r s t, s a+t b+f+s^{2} t\right) .
\end{aligned}
$$

Proof. Verifications are by direct calculation and are left to the reader.

Remark 10. The formulas are found by determining how the lines $y=m x+c$ and parabolas $y=(x-a)^{2}+b$ and $c=2(m-A)^{2}+B$ transform when the points are transformed as in (19). Then one only needs to check that the adjacencies between $V_{0}$ and $V_{2}$ and between $V_{1}$ and $V_{3}$ are preserved.

We note that the condition $t= \pm 1$ is needed in order to preserve the (vertical) 5cycles in $V_{0}$, and the condition $r^{2}=t$ is needed to preserve the family of parabolas $y=(x-a)^{2}+b,\left(a, b \in \mathbb{Z}_{5}\right)$, and thus the adjacencies between $V_{0}$ and $V_{3}$. After sections 5 and 6 we will see this in a different light: preservation of a family of maximum cocliques of $H_{1}$.

Remark 11. The square of the duality $\psi$ of $H$ introduced in $(4)$ is $(0, x, y) \stackrel{\psi^{2}}{\longmapsto}(0,3 x,-y)$, $(1, m, c) \stackrel{\psi^{2}}{\longmapsto}(1,3 m,-c)$ and satisfies the hypotheses of Theorem 2 . Therefore $\psi^{2}$ can be extended to an automorphism of $G$ : $(2, A, B) \stackrel{\psi^{2}}{\longmapsto}(2,3 A,-B),(3, a, b) \stackrel{\psi^{2}}{\longmapsto}(3,3 a,-b)$. Clearly, $\psi^{2}$ and its extension to $G$ have order 4 . It is not hard to find that $\psi$ itself can be extended to an automorphism of $G$ (of order 8) which interchanges $V_{0}$ with $V_{1}$ and $V_{2}$ with $V_{3}$ by defining $(2, A, B) \stackrel{\psi}{\longmapsto}(3,3 A, 2 B),(3, a, b) \stackrel{\psi}{\longmapsto}(2, a, 2 b)$. The automorphism $\psi^{4}$ is an involution whose fixed-point set of order 20 is the set $W_{0}$ defined in Section 12 .

The following Lemma introduces two further automorphisms which will allow us to show that $G$ is a Cayley graph (Theorem 2).

Lemma 12. Define mappings $g, h: G \rightarrow G$ by

$$
\begin{array}{lll|lll}
(0, x, y) & \stackrel{g}{\mapsto} & (0, x+1, y-x) & (0, x, y) & \stackrel{h}{\mapsto} & \left(1,2 x, 2 y-2 x^{2}\right) \\
(1, m, c) & \stackrel{g}{\mapsto} & (1, m-1, c-m+1) & (1, m, c) & \stackrel{h}{\mapsto} & \left(2, m, 2 c-2 m^{2}\right) \\
(2, A, B) & \stackrel{g}{\mapsto} & (2, A-2,-A+B-1) & (2, A, B) & \stackrel{h}{\mapsto} & (3,-A, 2 B) \\
(3, a, b) & \stackrel{g}{\mapsto} & (3, a-1,-a+b+1) & (3, a, b) & \stackrel{h}{\mapsto} & \left(0,2 a, 2 b+2 a^{2}\right)
\end{array}
$$

Then $g$ is an automorphism of order 5 which fixes each of the sets $V_{0}, \ldots, V_{3}$, and $h$ is an automorphism of order 4 of $G$ which cyclically permutes $V_{0}, \ldots, V_{3}$.

The proof is left as a computational exercise. The automorphism $h$ confirms our earlier observation that the four sides of the square in Fig. 2 are Hoffman-Singleton graphs. In conjunction with Theorem 2 it shows that $G$ is vertex transitive. The automorphism $g$ is an extension of an affine self-mapping of $V_{0}$ as described in Theorem 2. 
Remark 13. Considering the automorphism $h$ and its powers, we note that for $v \in$ $V_{i} \cup V_{i+1}$, the neighbours of $v$ in $V_{i+2} \cup V_{i+3}$ (subscripts modulo 4) form a coclique of order 15 in the Hoffman-Singleton subgraph induced in $G$ by $V_{i+2} \cup V_{i+3}$.

Theorem 3. (Heinze [21], Jørgensen-Klin [32], Praeger and Schneider [44]) The HigmanSims graph is a Cayley graph.

Proof. We can obtain a direct proof of this result from our explicit knowledge of the automorphisms $g$ and $h$. Note first that $g$ and $k=h^{-1} g h$ together generate an elementary abelian group of order 25 which acts transitively on $V_{0}$. (It may help to observe that $h^{-1} k h=g^{-1}$.) It is easy to see that $\langle g, h\rangle$ has order 100 and is a regular group of automorphisms of $G$. An abstract definition of this group by generators and relations as well as a suitable generator set are given on [15].

Remark 14. We note that, as a permutation, $h$ is product of 25 cycles of length 4 and hence an odd permutation. This implies that the automorphism group of the HigmanSims graph contains a subgroup of index 2, consisting of the automorphisms which are even permutations. This subgroup is the sporadic simple group HS.

Remark 15. Anticipating notation and results that will be introduced later, we note that an automorphism of $H_{1}$ can be extended to an automorphism of all of $G$ if and only if it preserves the families $\mathcal{F}_{1}$ and $\mathcal{F}_{2}$ of maximum cocliques of $H_{1}$.

\section{Maximum Cocliques in the Hoffman-Singleton Graph}

We will now derive a description of the maximum cocliques in the Hoffman-Singleton graph as sets of parabolas in the biaffine plane. It is well-known that maximum cocliques in the Hoffman-Singleton graph are of order 15; this can be proved via eigenvalues ([14], Theorem 2.12) or via an application of the Cauchy-Schwarz inequality $([31,13])$. It will also be a by-product of Lemma 16 .

In this section we will use geometric notation and terminology as much as possible. In particular, we will refer to the 'point vertices' $(0, x, y)$ of the Hoffman-Singleton graph as points $(x, y)$, and a 'line vertex' $(1, m, c)$ will be referred to as the line $y=m x+c$. We remind the reader that 'a vertical line' consists of vertices $(0, x, y)$ on a 5-cycle of $V_{0}$, with $x$ constant, $y \in \mathbb{Z}_{5}$ (and that vertical lines are not lines of our biaffine plane).

Lemma 16. A coclique of order $n \geq 15$ in the Hoffman-Singleton graph consists either of 5 points, one from each vertical line, and 5 pairs of non-adjacent parallel lines, or of 5 pairs of non-adjacent points on vertical lines and 5 lines, one from each parallel class. In particular, the order of a maximum coclique in the Hoffman-Singleton graph is 15.

Proof. Let $C$ be a coclique of order $n \geq 15$ in the Hoffman-Singleton graph. Since each parallel class of lines is a 5 -cycle, there can be at most two elements of each class in a coclique. In particular, $C$ must contain at least 5 points and at least 5 lines. 
Case 1: $C$ contains one pair of non-adjacent parallel lines. We may assume that they are the horizontal lines $y=0$ and $y=1$, since otherwise an adjacency-preserving affine transformation can bring us into this situation. Then none of the points $(x, 0)$ and $(x, 1)$, $x \in \mathbb{Z}_{5}$, can belong to $C$. Now $C$ contains at least 3 more lines, and we may assume that one of them is $y=m x,(m \neq 0)$-represented by the vertex $(1, m, 0)$ - otherwise we perform a translation $x \mapsto x+r$. The line $y=m x$ is incident with the points $(2 / m, 2)$ and $(4 / m, 4)$. Consequently, there can be at most one point in $C$ whose first coordinate is $2 / m$ because $(2 / m, 0),(2 / m, 1),(2 / m, 2)$ do not belong to $C$, and the points $(2 / m, 3)$ and $(2 / m, 4)$ are adjacent. Similarly, $C$ can contain only one point with first coordinate $4 / m$.

It follows that if $C$ contains a pair of non-adjacent parallel lines then $C$ contains at most 8 points, and therefore at least two pairs of non-adjacent parallel lines. By duality, the analogous statement with points and lines interchanged is also valid.

Case 2: $C$ contains two pairs of non-adjacent parallel lines, say $y=0, y=1, y=m x$ and $y=m x+1$. The line $y=m x$ meets the horizontal lines $y=0,1$ in $(0,0)$ and $(1 / m, 1)$ respectively. Then the line $y=m x+1$ passes through $(1 / m, 2)$, allowing only one of the adjacent points $(1 / m, 3)$ and $(1 / m, 4)$ to belong to $C$. Similarly, only one of the points on the vertical line $x=4 / m$ can belong to $C$. Our four lines have 4 distinct points of intersection with each of the vertical lines $x=2 / m$ and $x=3 / m$, so that $C$ cannot contain a pair of points from these vertical lines either.

It follows that if $C$ contains two non-adjacent parallel lines then $C$ can contain at most 1 pair of points from a vertical line. Since we established above that it is impossible for a maximum coclique to contain precisely one such pair of points, we conclude that if $C$ contains one pair of parallel lines then $C$ contains 5 pairs of parallel lines, but at most one point from each vertical line. By duality, if $C$ contains 2 points on a vertical line, then $C$ contains 5 such pairs and no pairs of parallel lines. In particular, we have established that the order of a maximum coclique in the Hoffman-Singleton graph is 15 .

Lemma 17. Let $C$ be a maximum coclique in the Hoffman-Singleton graph, and assume that $C$ consists of 5 points $p_{i}$ and 10 lines $\ell_{j}(i=1, \ldots, 5, j=1, \ldots, 10)$. Then no three of the points are collinear in the biaffine plane.

Proof. We note that the 10 lines must be partitioned into pairs of non-adjacent parallel lines. Assume that $p_{1}, p_{2}, p_{3}$ are collinear points of $C$, incident with the line $\ell$ with equation $y=0$. This is no loss of generality since we can always use an admissible affine transformation to transform a given line into $\ell$. Then the lines $y=m x+c(m \neq 0)$ in $C$ must pass through the remaining two points of $\ell$, say $\left(x_{4}, 0\right)$ and $\left(x_{5}, 0\right)$. If $y=m x+c_{1}$ and $y=m x+c_{2}$ are two such parallel lines, their adjacency is governed by the difference $c_{1}-c_{2}=m\left(x_{4}-x_{5}\right)$. There are exactly two values for $m$ which will make this a square and two which make it a non-square in $\mathbb{Z}_{5}^{*}$. This means that $C$ contains at most 3 pairs of non-adjacent lines, a contradiction.

Corollary 18. Assume that $C$ is a coclique in the Hoffman-Singleton graph consisting of 5 points and 10 lines. Then the 5 points form one of the sets with equation $y= \pm(x-a)^{2}+b$ in the biaffine plane. 
Proof. The 5 points together with the point of intersection of the vertical lines form an oval in the projective plane over $\mathbb{Z}_{5}^{*}$. By Segre's theorem [46], this is a conic. Since the line at infinity is a tangent, this conic is a parabola $y=r(x-a)^{2}+b, r \neq 0$ (in the affine plane). The 10 lines in the coclique represented by vertices $(1, m, c)$ are non-adjacent in the graph $H$ if and only if $r= \pm 1$, because the 3 values $(b, b+r, b-r)$ of $r(x-a)^{2}+b\left(x \in \mathbb{Z}_{5}\right)$ are consecutive mod 5 if and only if $r= \pm 1$. In that case, any pair of parallel lines which do not meet the parabola intersect a vertical line in adjacent points, and consequently the lines are non-adjacent in the graph (cf. the comment in the introduction).

Corollary 19. There are exactly 100 distinct cocliques of order 15 in the HoffmanSingleton graph.

Proof. We know from Corollary 18 that there are at most $50=2 \cdot 5 \cdot 5$ cocliques of order 15 containing exactly 5 points and 10 lines, and by duality there are at most 50 containing exactly 5 lines. It is an easy exercise to verify that the passants of the parabola $y=(x-a)^{2}+b$ are the 10 lines $y=m x+c$, where $c=m^{2}-m a+b \pm 2$; in other words: for any $a, b \in \mathbb{Z}_{5}$ the 15 vertices

$$
\begin{array}{ll}
\left(0, x,(x-a)^{2}+b\right), & x \in \mathbb{Z}_{5}, \\
\left(1, m, m^{2}-m a+b+2\right), & m \in \mathbb{Z}_{5}, \\
\left(1, m, m^{2}-m a+b-2\right), & m \in \mathbb{Z}_{5},
\end{array}
$$

form a 15-coclique in the Hoffman-Singleton graph. Similarly, the passants of the parabola $y=-(x-e)^{2}+f$ are given by the 10 lines $y=m x+c$ where $c=-m^{2}-m e+f \pm 2$.

Dually, the 5 lines $y=m x+c$ where $c=2(m-E)^{2}+F$ avoid all the points $(x, y)$ where $y=3 x^{2}+E x+F \pm 1$; and the 5 lines $y=m x+c$ where $c=3(m+E)^{2}-F$ avoid all the points $(x, y)$ where $y=2 x^{2}-E x-F \pm 1$.

This leads us to define the following 15-element sets for $a, b, A, B, e, f, E, F \in \mathbb{Z}_{5}$ :

$$
\begin{aligned}
P(a, b) & =\left\{\left(0, x,(x-a)^{2}+b\right): x \in \mathbb{Z}_{5}\right\} \cup\left\{\left(1, m, m^{2}-m a+b \pm 2\right): m \in \mathbb{Z}_{5}\right\}, \\
Q(A, B) & =\left\{\left(1, m, 2(m-A)^{2}+B\right): m \in \mathbb{Z}_{5}\right\} \cup\left\{\left(0, x, 3 x^{2}+A x+B \pm 1\right): x \in \mathbb{Z}_{5}\right\} \\
P^{\prime}(e, f) & =\left\{\left(0, x,-(x-e)^{2}+f\right): x \in \mathbb{Z}_{5}\right\} \cup\left\{\left(1, m,-m^{2}-m e+f \pm 2\right): m \in \mathbb{Z}_{5}\right\} \\
Q^{\prime}(E, F) & =\left\{\left(1, m, 3(m+E)^{2}-F\right): m \in \mathbb{Z}_{5}\right\} \cup\left\{\left(0, x, 2 x^{2}-E x-F \pm 1\right): x \in \mathbb{Z}_{5}\right\} .
\end{aligned}
$$

These sets $P(a, b)$ etc. are the 100 maximum cocliques of the Hoffman-Singleton graph, grouped into 4 sets of 25 . 


\section{Two Families of Maximum Cocliques and the Max- coclique Graph}

We define 2 families of 50 maximum cocliques of the Hoffman-Singleton graph as follows:

$$
\begin{aligned}
& \mathcal{F}_{1}=\left\{P(a, b): a, b \in \mathbb{Z}_{5}\right\} \cup\left\{Q(A, B): A, B \in \mathbb{Z}_{5}\right\}, \\
& \mathcal{F}_{2}=\left\{P^{\prime}(e, f): e, f \in \mathbb{Z}_{5}\right\} \cup\left\{Q^{\prime}(E, F): E, F \in \mathbb{Z}_{5}\right\} .
\end{aligned}
$$

These two families can be transformed into each other by means of the affine automorphism of $H$ induced by $(0, x, y) \mapsto(0, x,-y)$. Each of them is invariant under dualities of the Hoffman-Singleton graph. An intrinsic characterisation of these two families is obtained by looking at the cardinality of intersections of their members:

Lemma 20. Let $X \in \mathcal{F}_{1}$, and let $Y$ be any maximum coclique of the Hoffman-Singleton graph. Then

$$
\begin{aligned}
& Y \in \mathcal{F}_{1} \quad \text { if and only if } \quad|X \cap Y| \in\{0,5\} \text {, } \\
& Y \in \mathcal{F}_{2} \quad \text { if and only if } \quad|X \cap Y| \in\{3,8\} \text {. }
\end{aligned}
$$

The corresponding result with $\mathcal{F}_{1}$ and $\mathcal{F}_{2}$ interchanged also holds.

Proof. We give a sample calculation. Let $a, b, e, f \in \mathbb{Z}_{5}$ and consider the two cocliques $X=P(a, b)$ and $Y=P^{\prime}(e, f)$ (cf. (23) and (25)). One constructs two quadratic equations to determine their common points; after minimal manipulations they are:

$$
\begin{aligned}
2 x^{2}-2(e+a) x+a^{2}+e^{2}+b-f & =0, \\
2 m^{2}+m(e-a)+b-f+\nu+\mu & =0, \text { where } \nu, \mu \in\{2,-2\} .
\end{aligned}
$$

The discriminants are $\Delta_{1}=(a-e)^{2}+2(b-f)$ and $\Delta_{2}=(e-a)^{2}+2(b-f)+2(\nu+\mu)=$ $\Delta_{1}+2 \nu+2 \mu$, respectively.

It is impossible that $\Delta_{1}$ and $\Delta_{2}$ are both \pm 2 for all choices of $\nu, \mu$, hence $X$ and $Y$ cannot be disjoint.

When $\Delta_{1}= \pm 1$ then $X$ and $Y$ have 2 vertices of $V_{0}$ in common, and consideration of the possibilities for $\Delta_{2}$ reveals 3 combinations of $\mu, \nu$ such that $\Delta_{2}= \pm 1$ (and one combination yielding $\Delta_{2}= \pm 2$ ). Hence $X$ and $Y$ have $3 \cdot 2=6$ vertices of $V_{1}$ in common, for an intersection of cardinality 8 in total.

When $\Delta_{1}=0$ then $X$ and $Y$ have 1 common neighbour in $V_{0}$, and consideration of the possibilities for $\Delta_{2}$ reveals 2 combinations of $\mu, \nu$ such that $\Delta_{2}=0$ (and 2 combinations yielding $\Delta_{2}= \pm 2$ ). Hence $X$ and $Y$ have 2 vertices of $V_{1}$ in common for an intersection of cardinality 3 in total.

Remark 21. If one goes through all the detail of the preceding proof, the following stronger result is obtained: for given $X \in \mathcal{F}_{1}$ the number of maximum cocliques $Y$ with $|X \cap Y|=0,3,5,8,15$ is $7,35,42,15,1$, respectively. 
Theorem 4 (The max-coclique graph). Let $Z$ be the graph whose vertex set is $\mathcal{F}_{1} \cup$ $\mathcal{F}_{2}$ and adjacency is defined by disjointness. Then $\mathcal{F}_{1}$ and $\mathcal{F}_{2}$ each induce a connected component of $Z$, each of the components being isomorphic to the Hoffman-Singleton graph.

Proof. Lemma 20 and Remark 21 imply that there are two connected components. It remains to show that $\mathcal{F}_{1}$ induces a Hoffman-Singleton graph. The case for $\mathcal{F}_{2}$ then follows by applying the automorphism induced by the affine transformation $(0, x, y) \mapsto(0, x,-y)$. Thinking of the sets $P(a, b)$ and $Q(A, B)$ as unions of parabolas in the $(x, y)$ - and $(m, c)$ coordinate systems, it is obvious that $P(a, b) \cap P(r, s)=\emptyset$ if and only if $r=a$ and $s=b \pm 2$. Similarly, $Q(A, B) \cap Q(R, S)=\emptyset$ if and only if $R=A$ and $S=B \pm 1$. Next we observe that $P(a, b) \cap Q(A, B)=\emptyset$ if and only if none of the following 4 equations (in $x$ or $m$ ) has a solution in $\mathbb{Z}_{5}$ :

$$
\begin{aligned}
(x-a)^{2}+b & =3 x^{2}+A x+B \pm 1, \\
2(m-A)^{2}+B & =m^{2}-a m+b \pm 2 .
\end{aligned}
$$

The discriminants of these equations equal \pm 2 if and only if $B=2 A^{2}+3 a A-a^{2}+b$.

Now we see that $\mathcal{F}_{1}$ induces a component which is isomorphic to the subgraph of $G$ induced by $V_{2} \cup V_{3}$ : just identify the cocliques $P(a, b), Q(A, B)$ with the vertices $(3, a, b)$ and $(2, A, B)$ of $G$, respectively. We have seen in Remark 8 that this graph is isomorphic to the Hoffman-Singleton graph.

Corollary 22. (1) The graph $G$ is isomorphic to the graph whose vertex set is $H \cup \mathcal{F}_{1}$, where adjacency in $H$ is defined as in Definition 1 , adjacency in $\mathcal{F}_{1}$ is disjointness of cocliques, and $v \in H$ is adjacent to $X \in \mathcal{F}_{1}$ if and only if $v \in X$.

(2) The graph $G$ is isomorphic to the graph whose vertex set is $H \cup \mathcal{F}_{2}$, where adjacency in $H$ is defined as in Definition 1 , adjacency in $\mathcal{F}_{2}$ is disjointness of cocliques, and $v \in H$ is adjacent to $X \in \mathcal{F}_{2}$ if and only if $v \in X$.

(3) Each coclique in $\mathcal{F}_{i}, i \in\{1,2\}$, contains 15 vertices of $H$, and each vertex of $H$ is contained in 15 cocliques of $\mathcal{F}_{i}$.

Proof. For (1), recall the definition of $\mathcal{F}_{1}$ (cf. (27)) in equations (23) and (24), and note that membership of $(0, x, y)$ or $(1, m, c)$ in a coclique of $\mathcal{F}_{1}$ is governed by the same equations as those defining adjacency between vertices of $V_{0} \cup V_{1}$ and $V_{2} \cup V_{3}$ in $G$ (cf. Fig. 2 and equations (7)-(16)). Adjacency between vertices of $\left\{P(a, b): a, b \in \mathbb{Z}_{5}\right\}$ and $\left\{Q(A, B): A, B \in \mathbb{Z}_{5}\right\}$ was shown in the proof of Theorem 4 to be governed by the same equations that apply in $V_{2} \cup V_{3}$. The reader will have no difficulty confirming the 5-cycle adjacencies (labelled \pm 1 and \pm 2 in Fig. 2) within $\left\{Q(A, B): A, B \in \mathbb{Z}_{5}\right\}$ and $\left\{P(a, b): a, b \in \mathbb{Z}_{5}\right\}$.

(2) Application of the automorphism of $H$ induced by $(0, x, y) \mapsto(0, x,-y)$ maps $\mathcal{F}_{1}$ to $\mathcal{F}_{2}$ and preserves disjointness of cocliques and incidence of vertices with cocliques.

(3) One part of this statement is evident from the definition of $\mathcal{F}_{i}$, and the other follows by applying the automorphism $h^{2}$ (cf. Lemma 12), bearing in mind Theorem 4 . 
Remark 23. It follows from James [30] that every 5-cycle of the Hoffman-Singleton graph $H_{1}$ determines a split of $H_{1}$ into a pair of $5 C_{5}$, and that whenever $\left\{V_{0}^{\prime}, V_{1}^{\prime}\right\}$ is a split of $H_{1}$ into a pair of $5 C_{5}$ then there exists an automorphism of $H_{1}$ mapping $V_{0}$ to $V_{0}^{\prime}$ and $V_{1}$ to $V_{1}^{\prime}$. This implies that given a 15-coclique $C$ of $H_{1}$ and any split of $H_{1}$ into a pair $\left\{V_{0}^{\prime}, V_{1}^{\prime}\right\}$ of $5 C_{5}$, the number of elements of $C$ in $V_{0}^{\prime}$ will be 5 or 10 ; and the intersection of $C$ with any 5 -cycle of $H_{1}$ will consist of 1 or 2 vertices.

Corollary 22 shows that there is not only an automorphism of $H_{1}$ but also of $G$ which maps $V_{0}$ to $V_{0}^{\prime}$ and $V_{1}$ to $V_{1}^{\prime}$ (because $V_{2}$ and $V_{3}$ are identified with cocliques in $\mathcal{F}_{1}$ and adjacencies determined by membership and disjointness).

Remark 24. Corollary 22 characterises the cocliques of $\mathcal{F}_{1}$ as the sets of neighbours in $H_{1}$ of vertices in $H_{2}$. Application of $h$ and its powers shows more generally that if $v \in V_{i} \cup V_{i+1}$ then the neighbours of $v$ in $V_{i+2} \cup V_{i+3}$ (indices mod 4) form a coclique in the Hoffman-Singleton subgraph induced by $V_{i+2} \cup V_{i+3}$; i.e. edges between $V_{i} \cup V_{i+1}$ and $V_{i+2} \cup V_{i+3}$ correspond to incidence of vertices of a Hoffman-Singleton subgraph and its maximum cocliques.

Remark 25. We now look back at Theorem 1 in the light of Corollary 22. To see that $G$ is triangle-free, consider three vertices $v_{1}, v_{2}, v_{3}$ of $G$. If all three are contained in $H_{1}$ or in its complement $H_{2}$, they cannot form a triangle because the Hoffman-Singleton graph has girth 5. If not all three belong to $H_{1}$ or to $H_{2}$, we may assume that $v_{1} \in H_{1}$, $v_{2}, v_{3} \in H_{2}$ (apply the automorphism $h^{2}$ if need be). If $v_{2}$ and $v_{3}$ are adjacent, then their neighbourhoods in $H_{1}$ are disjoint cocliques, which means that $v_{1}$ cannot belong to both of them.

To see that any two non-adjacent vertices $v_{1}, v_{2}$ of $G$ have exactly 6 common neighbours, assume first that both vertices belong to $H_{1}$. There is a unique common neighbour $v$ in $H_{1}$, and we may assume that $v_{1}, v, v_{2}$ belong to a vertical 5 -cycle. Now our knowledge of maximum cocliques makes it evident that there are exactly 5 of them which contain $v_{1}$ and $v_{2}$ (i.e. there are exactly 5 common neighbours of $v_{1}$ and $v_{2}$ in $H_{2}$ ). If, secondly, $v_{1} \in H_{1}, v_{2} \in H_{2}$, we prove the following result (the first part is contained in Neumaier's Proposition 3 or Jeurissen's Lemma 6.2) by a simple calculation.

Lemma 26. (Neumaier [38], Jeurissen [31]) Let $v$ be a vertex of the Hoffman-Singleton graph and $C$ a maximum coclique in $\mathcal{F}_{1}$ not containing $v$.

(1) There exist exactly 3 cocliques in $\mathcal{F}_{1}$ which contain $v$ and which are disjoint from $C$.

(2) There are exactly 3 vertices in $C$ which are adjacent to $v$.

The same is true if $\mathcal{F}_{1}$ is replaced by $\mathcal{F}_{2}$ throughout.

Proof. We first consider the case where $v=(0, x, y) \in V_{0}$, assuming that $C$ consists of all neighbours in $H_{1}$ of $w=(2, A, B) \in V_{2}$, with $y=3 x^{2}+A x+B+\delta$ and $\delta \in\{0, \pm 2\}$ (non-adjacency). If $\delta=0$, then $v$ and $C$ have two common neighbours $(2, A, B \pm 1)$ in $V_{2}$, and otherwise only one. Any further common neighbours in $V_{2} \cup V_{3}$ must belong to $V_{3}$ and satisfy the equation

$$
-x^{2}+2 a x-a^{2}+y=B-2 A^{2}+2 a A+a^{2}
$$


(refer to Fig. 2). Substitute $y=3 x^{2}+A x+B+\delta$ into this quadratic equation for $a$, and note that its discriminant is $-2 \delta$. This shows that there are 1 resp. 2 further neighbours common to $v$ and $w$ in $H_{2}$, according to whether $\delta=0$ or $\delta= \pm 2$. In the language of maximum cocliques of $H_{1}$ this means that there are exactly three cocliques of $\mathcal{F}_{1}$ which contain $v$ and which are disjoint from $C$.

The proof of (1) in the case where $v=(0, x, y) \in V_{0}, w=(3, a, b) \in V_{3}$, with $y=(x-a)^{2}+b+\delta$ and $\delta \neq 0$ is similar: if $\delta= \pm 1$ then $v$ and $w$ have no common neighbours in $V_{3}$, and if $\delta= \pm 2, v$ and $w$ have 1 common neighbour in $V_{3}$. Vertices $(2, A, B)$ which are adjacent to $v$ as well as $w$ must satisfy the equation

$$
(x-a)^{2}+b+\delta=3 x^{2}+A x+B \pm 1
$$

where $B=2 A^{2}+3 a A-a^{2}+b$. The discriminant of the resulting quadratic equation for $A$ is $3(\delta \pm 1)$. This shows that there are 3 solutions in the case when $\delta= \pm 1$ and 2 solutions in the case when $\delta= \pm 2$, so that in each case we have 3 common neighbours of $v$ and $w$ in $\mathrm{H}_{2}$.

To see that part (2) is merely a 'dual' of part (1), consider the Hoffman-Singleton graph $H_{1}$ in $G$. The vertex $v$ has 15 neighbours in $H_{2}$, forming a coclique $D$ (and each vertex of $D$ representing a coclique in $H_{1}$ ). The coclique $C$ in $H_{1}$ consists of all neighbours in $H_{1}$ of a vertex $w \in H_{2}$. Now apply part (1) in $H_{2}$ : there exist precisely 3 vertices in $D$ which are adjacent to $w$. These three vertices represent cocliques containing $v$ which are disjoint from $C$.

To get the same results for $C \in \mathcal{F}_{2}$, we extend the affine mapping $(0, x, y) \mapsto(0, x,-y)$ to an automorphism of $H$ which swaps $\mathcal{F}_{1}$ and $\mathcal{F}_{2}$.

Remark 27. The previous lemma looks very much like a parallel axiom in a geometry made up of the vertices of $H$ as points and maximum cocliques from $\mathcal{F}_{1}$ as blocks. This incidence structure, a partial 5-geometry, was first observed by Neumaier [38]. In the terminology of [28] it is a semisymmetric design $D$ with parameters $(50,15,[5])$ while [35] speaks of an $\operatorname{SPBIBD}(50,15 ; 0,5)$. We note that [38] has a realisation of the incidence graph of this design in the Leech lattice, but also describes it in terms of the 100 maximum cocliques in the Hoffman-Singleton graph.

Remark 28. Here is another approach to constructing the Higman-Sims graph: let $T$ be the incidence graph of the semisymmetric design $D$ defined in Remark 27 (whose automorphism group $A$ is isomorphic to the automorphism group of the Hoffman-Singleton graph); now add one of the edges $\{x, y\}$ of $H$ as well as all the images of $\{x, y\}$ under $A$. The result is the Higman-Sims graph - the new edges producing a vast increase in the order of the automorphism group.

For future reference, we include the following simple result about vertex stabilisers without proof.

Lemma 29. Let $H$ be the Hoffman-Singleton graph and denote by $A u t^{+}(H)$ the subgroup of $A$ ut $(H)$ consisting of those automorphisms which preserve the two families $\mathcal{F}_{1}$ and $\mathcal{F}_{2}$. 


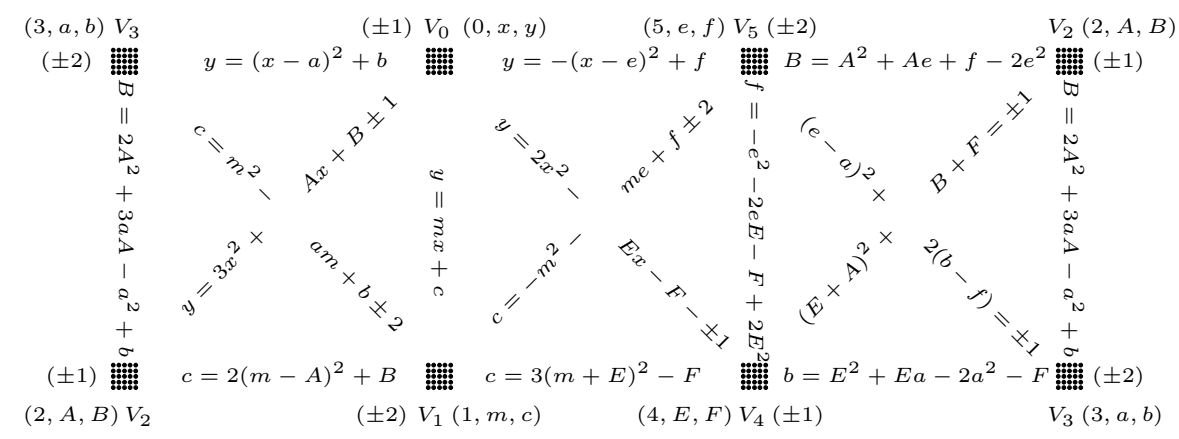

Figure 3: The supergraph $K$ (note the wraparound, and twist)

1. The stabiliser of a vertex of $H$ in $A u t(H)$ is the symmetric group $S_{7}$ in its natural action on the neighbours of the vertex.

2. The stabiliser of a vertex of $H$ in $A u t^{+}(H)$ is the alternating group $A_{7}$.

\section{The Supergraph}

We will now give an explicit construction of a 'supergraph' $K$ of order 150, constructed from 3 Hoffman-Singleton graphs which are linked cyclically, so that removal of any one of them produces a graph isomorphic to $G$. This graph $K$ is mentioned in [5], p.108, [6], p. 394. It provides an ideal environment for the study of maximum cocliques in the Hoffman-Singleton graph (and therefore for the study of the Higman-Sims graph), as we shall see.

The vertex set of the graph $K$ is $V_{0} \cup \cdots \cup V_{5}$, with adjacencies on $V_{0} \cup \cdots \cup V_{3}$ defined as before (vertices in $V_{2} \cup V_{3}$ representing 15-cocliques of $\mathcal{F}_{1}$ ). A second Higman-Sims graph is constructed on $V_{0} \cup V_{1} \cup V_{4} \cup V_{5}$ (vertices in $V_{4} \cup V_{5}$ representing 15-cocliques of $\mathcal{F}_{2}$ ), using equations (25) and (26). Finally, we define vertices of $u \in V_{2} \cup V_{3}$ and $v \in V_{4} \cup V_{5}$ to be adjacent when they have 8 common neighbours in $H_{1}$ (i.e. when their corresponding 15 -cocliques intersect in 8 vertices). The resulting graph is described in Fig. 3; note that the figure wraps around, but a twist is needed when identifying the left and right. To see that the graph induced by $V_{2} \cup \cdots \cup V_{5}$ is isomorphic to the HigmanSims graph $G$, one shows that the neighbours of vertices in $H_{2}$ form 15 -cocliques in $H_{3}$, and that cocliques corresponding to adjacent vertices of $H_{2}$ are disjoint. We omit the calculations. $\left(H_{1}, H_{2}, H_{3}\right.$ are the subgraphs of order 50 induced by $V_{0} \cup V_{1}, V_{2} \cup V_{3}$, and $V_{4} \cup V_{5}$ respectively. All three are isomorphic to the Hoffman-Singleton graph.)

Remark 30. In the graph $G$, only those automorphisms of $H_{1}$ which preserve the families $\mathcal{F}_{1}, \mathcal{F}_{2}$ can be extended to an automorphism of $G$. By contrast, every automorphisms of $H_{1}$ can be extended to an automorphism of $K$. Those automorphisms of $H_{1}$ which interchange $\mathcal{F}_{1}$ and $\mathcal{F}_{2}$ will swap $H_{2}$ and $H_{3}$. The full group of automorphisms of $K$ has order $3 \cdot 252000=756000$. 


\section{Hoffman-Singleton Subgraphs of $G$}

In this section we study the Hoffman-Singleton subgraphs of the Higman-Sims graph $G$. The structures revealed in the process will be discussed further in Section 9. Lemmas 31 and 32 show that $\operatorname{Aut}(G)$ is transitive on Hoffman-Singleton subgraphs; but there are two orbits under the action of the subgroup HS (which consists of the even permutations amongst the automorphisms).

Lemma 31. Let $Z$ be an $\operatorname{srg}(100,22,0,6)$ and assume that $X$ is an induced HoffmanSingleton subgraph of $Z$. Then $Y=Z \backslash X$ is also a Hoffman-Singleton graph. The neighbours in $X$ of a vertex $v \in Y$ form maximum cocliques in $X$ which intersect in 0 or 5 vertices. Therefore they all belong to the same family of maximum cocliques of $X$ and hence there exists an automorphism of $Z$ mapping $X$ to $Y$.

Proof. The statement of the lemma gives sufficient indication of the proof. We note that the lemma is a slight modification of Exercise 2 in [8], p. 113, but with a different approach to the proof.

Lemma 32. Let $X, Y$ be Hoffman-Singleton subgraphs of $G$. If $\tau \in A$ ut $(G)$ is an even permutation of degree 100 such that $X^{\tau}=Y$ then all automorphisms of $G$ which map $X$ to $Y$ are even.

Proof. Let $\sigma \in \operatorname{Aut}(G)$ be another automorphism of $G$ with $X^{\sigma}=Y$. Then $\sigma \tau^{-1}$ belongs to the stabiliser of $X$ in $\operatorname{Aut}(G)$, which is a simple group (cf. Remark 49) and therefore contains only even permutations.

Theorem 5. The graph $G$ contains exactly 704 Hoffman-Singleton subgraphs.

We split the proof into a sequence of lemmas which at the same time will help us become more familiar with the graphs $G$ and $K$.

The following lemma allows the reconstruction of the Higman-Sims graph as in Fig. 2, starting from a 5 -cycle. It is stated without proof.

Lemma 33. Let $F=\left\{v_{0}, \ldots, v_{4}\right\}$ be a 5-cycle in $V_{0}$, where $v_{i}$ is adjacent to $v_{i \pm 1}$ (subscripts modulo 5). Then $V_{2}$ is the union of the sets of common neighbours outside $F$ of $v_{i}$ and $v_{i+2}, i=0, \ldots, 4$.

Lemma 34. Let $\tau$ be an automorphism of $G$ which preserves $H_{1}$ and $H_{2}$. Then $\left|V_{3}^{\tau} \cap V_{3}\right|=$ $\left|V_{0}^{\tau} \cap V_{0}\right|$.

Proof. If $V_{0}^{\tau}=V_{0}$ or $V_{1}$, the statement is obvious. Assume therefore that $\left|V_{0}^{\tau} \cap V_{0}\right|=15$ (cf. Remark 1; the case where this intersection contains 10 vertices follows by duality). In view of Lemma 33 we find $V_{0}^{\tau}$ by choosing a 5-cycle $F$ in $V_{0}^{\tau}$ and collecting the common neighbours outside $F$ of vertices at distance 2 in $F$. Now we show that each 5-cycle of $V_{2}^{\tau}$ has 2 vertices in $V_{2}$. To this end, consider the case when $\tau$ maps some 5 -cycle of $V_{0}$ to

$$
(0,0,0),(0,0,1),(0,0,2),(1,0,2),(1,0,0) \text {. }
$$




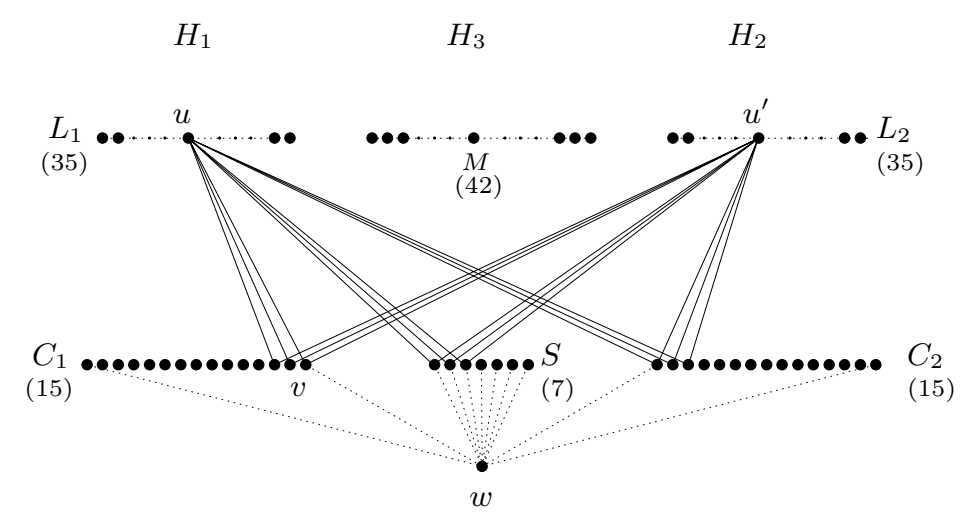

Figure 4: The graph $K$, as seen from the vertex $w$.

All other cases follow from this one by extending affine automorphisms of $H_{1}$ which fix $V_{0}$. Applying Lemma 33 one finds readily that one of the 5-cycles in $V_{2}^{\tau}$ is

$$
(2,0,0),(2,0,1),(2,0,2),(3,0,2),(3,0,0) .
$$

Therefore $\left|V_{2}^{\tau} \cap V_{2}\right|=15$ and hence $\left|V_{3}^{\tau} \cap V_{3}\right|=15=\left|V_{0}^{\tau} \cap V_{0}\right|$.

Lemma 35. There are (at least) 125 pairs $\left\{X_{1}, X_{2}\right\}$ of Hoffman-Singleton subgraphs of $G$ such that

$$
X_{1} \cup X_{2}=G, \quad\left|X_{1} \cap V_{0}\right|=\left|X_{1} \cap V_{3}\right|=15, \quad \text { and } \quad\left|X_{1} \cap V_{1}\right|=\left|X_{1} \cap V_{2}\right|=10 .
$$

Proof. By Remark 23 there exists an automorphism $\tau$ of $G$ such that $V_{0}^{\tau}=V_{0}^{\prime}$, and $V_{1}^{\tau}=V_{1}^{\prime}$. By Lemma 34, $X_{1}=V_{3}^{\tau} \cup V_{0}^{\tau}$ and $X_{2}=V_{2}^{\tau} \cup V_{3}^{\tau}$ have the required properties. Since there are 125 splits of $H_{1}$ into pairs of $5 C_{5}$ other than $\left\{V_{0}, V_{1}\right\}$, we have the numerical result. We add that our final census (after Lemma 39) of Hoffman-Singleton subgraphs will show that the estimate of 125 pairs with the desired properties is sharp (hence the parentheses).

Together with the 2 Hoffman-Singleton subgraphs $H_{1}$ and $H_{2}$, Lemma 35 produces 252 Hoffman-Singleton subgraphs. A further 252 of them are obtained by applying the automorphism $h$. To find another 200 Hoffman-Singleton subgraphs, we consider Fig. 4. It shows the supergraph $K$ as seen from a vertex $w \in H_{3}$; the graph $H_{3}$ appears as the Moore graph of degree 7 , with $S$ as neighbours of $w$. The remaining $30=15+15$ neighbours of $w$ form maximum cocliques $C_{1} \subset H_{1}$ and $C_{2} \subset H_{2}\left(C_{1}\right.$ is an $\mathcal{F}_{2}$-coclique). The (set-theoretic) complements of these cocliques in $H_{1}$ and $H_{2}$ respectively are $L_{1}$ and $L_{2}$. Note that for $i=1,2$ the subgraph $S \cup C_{i}$ is a coclique, since $H_{i} \cup H_{3}$ induces a Higman-Sims graph and thus is triangle-free.

Lemma 36. For $u^{\prime} \in L_{2}$ denote by $N\left(u^{\prime}\right)$ the set of neighbours of $u^{\prime}$ in $S$. Let $T$ be the set of triples of elements in $S$. Then

$$
u^{\prime} \stackrel{\phi}{\mapsto} N\left(u^{\prime}\right)
$$


determines a bijection $\phi$ between $L_{2}$ and $T$. A similar bijection can be defined between $L_{1}$ and $T$.

Proof. Consider a vertex $u^{\prime} \in L_{2}$. Since the subgraph of $K$ induced by $H_{2} \cup H_{3}$ is isomorphic to $G$ and therefore an $\operatorname{srg}(100,22,0,6)$ (Theorem 1), the vertices $u^{\prime}$ and $w$ have 6 neighbours in common, with exactly 3 of them in $C_{2}$ by Lemma 26. Consequently, $u^{\prime}$ has precisely 3 neighbours in $S$.

By Remark 30, if $\tau$ is an automorphism of $H_{3}$ which fixes $w$ and which preserves the two families of maximum cocliques of $H_{3}$ then $\tau$ can be extended to an automorphism of $K$ which maps $H_{1}$ to $H_{1}$ and $H_{2}$ to $H_{2}$. The restriction of $\tau$ to $S$ is $A_{7}$ (Lemma 29), and therefore transitive on triples of elements of $S$. Since there are 35 elements of $L_{2}$ and $\left(\begin{array}{l}7 \\ 3\end{array}\right)=35$ triples of elements of $S$, the Higman-Sims graph induced by $H_{2} \cup H_{3}$ in $K$ provides an explicit representation of a one-to-one correspondence between $L_{2}$ and triples from $S$. The same reasoning applies to $L_{1}$.

Remark 37. We can explore the action of $A_{7}$ on $S$ a little further, considering orbits of pairs of triples of elements of $S$ (which correspond to edges or non-edges in $L_{2}$ ). One counts 70 pairs of disjoint triples, 315 pairs of triples which intersect in one point, and 210 pairs of triples which intersect in two points. Since the subgraph induced by $L_{2}$ is regular of degree 4 , and therefore has 70 edges, we conclude that two vertices of $L_{2}$ are adjacent precisely then when their corresponding triples in $S$ are disjoint (edges must correspond to a union of orbits of $A_{7}$ on pairs of triples from $S$, since $A_{7}$ acts as a group of automorphisms of $L_{2}$ ).

We anticipate Lemma 43 which identifies $L_{2}$ with the set of lines of PG(3,2) and note that $\mathrm{PG}(3,2)$ contains 315 pairs of intersecting lines. Our list of orbit cardinalities for pairs of triples of elements from $S$ now implies that the bijection $\phi$ associates intersecting lines with triples having exactly one point in common. In other words: this famous correspondence [20] is hardwired into the Higman-Sims graph.

Composing the two bijections from Lemma 36 we obtain a bijection $u \leftrightarrow u^{\prime}$ between $L_{1}$ and $L_{2}$ which we introduce formally in the following definition.

Definition 3. For $u \in L_{1}$ we define $u^{\prime} \in L_{2}$ to be the vertex which has the same 3 neighbours in $S$ as $u$.

Lemma 38. Let $u \in L_{1}$. Then $u$ and $u^{\prime}$ have the same 3 neighbours in each of $C_{1}$, $S$, and $C_{2}$. Moreover, if $z \in L_{1}$, then $u$ and $z^{\prime}$ are adjacent if and only if $u^{\prime}$ and $z$ are adjacent.

Proof. Let $w=(4,0,0)$ and consider 3 of its neighbours in $H_{3}$, say $v_{1}=(4,0,-1), v_{2}=$ $(4,0,1), v_{3}=(5,0,0)$ (any three neighbours will do, but they can always be transformed into these by an automorphism of $K$ fixing $w$, since $A_{7}$ is transitive on triples from $S$ ). One finds easily that $u=(0,0,0)$ and $u^{\prime}=(2,0,0)$ are the two vertices of $L_{1}$ resp. $L_{2}$ having $v_{1}, v_{2}, v_{3}$ as common neighbours. As one calculates the three neighbours of $u, u^{\prime}$ in $C_{1}, C_{2}$ respectively, it turns out that $(0,0,-1),(0,0,1),(1,0,0)$ and $(2,0,-1),(2,0,1),(3,0,0)$ are common neighbours of $u$ and $u^{\prime}$, and that there are no other edges between any of the vertices considered. 
Now we let $A_{7}$ operate. $C_{1}$ and $C_{2}$ are invariant, the orbit of $u$ is $L_{1}$, and $u$ and $u^{\prime}$ are not adjacent. Therefore there is never an edge between a vertex $x \in L_{1}$ and its counterpart $x^{\prime} \in L_{2}$, and $x$ and $x^{\prime}$ always share the same neighbours in $C_{1}$ and in $C_{2}$. In addition, observe that for any $u, z \in L_{1}$ there is an edge between $u$ and $z^{\prime}$ if and only if there is an edge between $u^{\prime}$ and $z$ (an even permutation of $S$ which swaps the triples corresponding to $u$ and $z$ can be extended to an automorphism of $K$ which must interchange $u$ and $z$, as well as $u^{\prime}$ and $z^{\prime}$, preserving the presence or absence of any edges).

When considering the graph $G=H_{1} \cup H_{2}$, we can think of it embedded in the supergraph $K$. The neighbours of $w \in H_{3}$ form an $\mathcal{F}_{2}$-coclique $C_{1}$ in $H_{1}$, together with a maximum coclique $C_{2}$ of $H_{2}$. These two cocliques are paired in a natural way: each vertex of $C_{2}$ has precisely 8 neighbours in $C_{1}$, and each vertex in $C_{1}$ has precisely 8 neighbours in $C_{2}$ (cf. Lemma 20 -note that the neighbours in $H_{1}$ of a vertex in $H_{2}$ form an $\mathcal{F}_{1}$-coclique).

Lemma 39. Let $C_{1}$ be an $\mathcal{F}_{2}$-coclique in $H_{1}, w \in H_{3}$ the corresponding vertex of $H_{3}$, and $\mathrm{C}_{2}$ the associated maximum coclique of $\mathrm{H}_{2}$. Then the mapping $\tau: G \rightarrow G$ which interchanges $u$ and $u^{\prime}$ for all $u \in L_{1}$ and fixes each vertex of $C_{1}$ and of $C_{2}$ is an automorphism of $G$. Moreover, $\tau$ is an odd permutation, hence $\tau \notin H S$.

Proof. This follows immediately from Lemma 38: adjacencies between $L_{1}$ resp. $L_{1}^{\tau}$ and $C_{1}, C_{2}$ are unaffected, and $\tau$ is compatible with adjacencies between $L_{1}$ and $L_{2}$.

Proof of Theorem 5. We have seen that $L_{2} \cup C_{1}$ is a Hoffman-Singleton subgraph of $G$. Since there are 50 possibilities to choose $w$, and hence 50 possibilities to choose $C_{1}$ and $C_{2}$, we have found 50 more Hoffman-Singleton graphs. They are all distinct from the 504 which we have already, since the intersections with $V_{0}, V_{1}$ have cardinalities 5,10 or 10,5 respectively $\left(C_{1}\right.$, like any 15 -coclique, meets $V_{0}$ in 5 or 10 vertices and $V_{1}$ in 10 or 5). Now apply $h, h^{2}, h^{3}$ to finally obtain the total of 704 Hoffman-Singleton subgraphs. The next two Lemmas show that there are no further Hoffman-Singleton subgraphs: the total number of 5-cycles in $G$ is 443520 (Lemma 41), each contained in two HoffmanSingleton subgraphs. Therefore the number of Hoffman-Singleton subgraphs is at most $2 \cdot 443520 / 1260=704$.

Lemma 40. Let $Z$ be an $\operatorname{srg}(100,22,0,6)$ and let $F$ be a 5-cycle of $Z$. Then $F$ is contained in 0 or 2 Hoffman-Singleton subgraphs of $Z$.

Proof. Assume that $Z$ contains a Hoffman-Singleton subgraph $H$ which contains $F$. In view of Lemma 31 we may assume that $Z=G$, that $H$ is the subgraph induced by $V_{0} \cup V_{1}$, and that $F$ is one of the five 5-cycles in $V_{0}$ (otherwise we apply an automorphism of $H$ which can be extended to $G$ ). Then it is clear that $F$ belongs to two HoffmanSingleton subgraphs, namely to $H$ and also to the subgraph induced by $V_{0} \cup V_{3}$. To see that there are no other Hoffman-Singleton subgraphs containing $F$, we note first that every vertex of $G$ outside $V_{0}$ has a neighbour in $F$, and each vertex of $V_{2}$ has 2 neighbours in $F$. The latter means that no vertex of $V_{2}$ can be part of a Hoffman-Singleton graph $X$ containing $F$; the former shows that $X$ must contain $V_{0}$, since $X$ must contain four 5 -cycles without neighbours in $F$. Additional 5-cycles of $X$ which are disjoint from $V_{0}$ 
must therefore consist of vertices in $V_{1} \cup V_{3}$ and must contain at least one edge $\{u, v\}$ from $V_{1}$ (without loss of generality). Since each 5-cycle in $V_{3}$ contains 4 neighbours of this edge, it is impossible to construct five disjoint 5-cycles forming a $5 C_{5}$ which are not entirely contained in $V_{1}$ or $V_{3}$.

Lemma 41. Assume that $Z$ is a strongly regular graph with parameters $(100,22,0,6)$.

1. $Z$ contains 443520 pentagons, 22176 through each vertex.

2. Z contains 28875 4-cycles, 1155 through each vertex.

Proof. Let $v$ be a vertex of $Z$. To count the 5-cycles through $v$, choose two neighbours of $v, a$ and $b$. Since $Z$ is triangle-free, there are exactly 6 neighbours of $a$ which are also adjacent to $b$ : these must be avoided, otherwise the pentagon $v, a, x, y, b$ contains a triangle $x, y, a$. That leaves $22-6=16$ neighbours of $a$ which are not adjacent to $b$, each of which allows 6 ways to finish off a pentagon. Hence there are $\left(\begin{array}{c}22 \\ 2\end{array}\right) \cdot 16 \cdot 6=22176$ pentagons through a given vertex $v$. The total number of pentagons in $Z$ is therefore $22176 \cdot 100 / 5=443520$.

Now we count the 4-cycles. Any two non-adjacent vertices in $Z$ have 6 common neighbours. Choosing two of these neighbours determines a 4-cycle with the given nonedge as diagonal. $Z$ is regular of degree 22 , and therefore has 1100 edges and $\left(\begin{array}{c}100 \\ 2\end{array}\right) / 2-$ $1100=3850$ non-edges. Each of these non-edges is a diagonal in $\left(\begin{array}{l}6 \\ 2\end{array}\right)=154$-cycles. As each 4 -cycle has two diagonals, we find that the total number of 4-cycles is $3850 \cdot 15 / 2=28875$ (or 1155 such cycles through each vertex).

To conclude this section, we list the intersections of the 704 Hoffman-Singleton subgraphs of $G$ with the sets $V_{0}, \ldots, V_{3}$.

Lemma 42. The following table lists the cardinalities of the intersections of the 704 Hoffman-Singleton subgraphs of $G$ with the sets $V_{0}, \ldots, V_{3}$. Rows 1 and 2 belong to one of the HS-orbits, rows 3 and 4 to the other. The last row lists the number of Hoffman-Singleton graphs with each intersection pattern above it. It is evident that members $X$ of the $H S$-orbit of $H_{1}$ are characterised by the fact that $\left(\left|X \cap H_{1}\right|, \mid X \cap\right.$ $\left.H_{2} \mid\right) \in\{(20,30),(30,20),(0,50),(50,0)\}$, whilst the corresponding cardinalities are 
$(15,35),(35,15),(25,25)$ for the other HS-orbit.

\begin{tabular}{|c|c|c|c|c|}
\hline & (a) & (b) & $(c)$ & $(d)$ \\
\hline \begin{tabular}{|l|l|}
$V_{3}$ & $V_{0}$ \\
\end{tabular} & \begin{tabular}{|l|l|}
15 & 10 \\
\end{tabular} & \begin{tabular}{|l|l|}
10 & 5 \\
\end{tabular} & \begin{tabular}{|l|l|}
20 & 15 \\
\end{tabular} & \begin{tabular}{|l|l|}
0 & 25 \\
\end{tabular} \\
\hline \begin{tabular}{|l|l|}
$V_{2}$ & $V_{1}$ \\
\end{tabular} & \begin{tabular}{|l|l|}
15 & 10 \\
\end{tabular} & \begin{tabular}{|l|l|}
20 & 15 \\
\end{tabular} & \begin{tabular}{|l|l|}
10 & 5 \\
\end{tabular} & \begin{tabular}{|l|l|}
0 & 25 \\
\end{tabular} \\
\hline \begin{tabular}{|l|l|}
$V_{3}$ & $V_{0}$ \\
\end{tabular} & \begin{tabular}{|l|l|}
10 & 15 \\
\end{tabular} & \begin{tabular}{|l|l|}
15 & 20 \\
\end{tabular} & \begin{tabular}{|l|l|}
5 & 10 \\
\end{tabular} & \begin{tabular}{|l|l|}
25 & 0 \\
\end{tabular} \\
\hline \begin{tabular}{|l|l|}
$V_{2}$ & $V_{1}$ \\
\end{tabular} & \begin{tabular}{|l|l|}
10 & 15 \\
\end{tabular} & \begin{tabular}{|l|l|}
5 & 10 \\
\end{tabular} & \begin{tabular}{|l|l|}
15 & 20 \\
\end{tabular} & \begin{tabular}{|l|l|}
25 & 0 \\
\end{tabular} \\
\hline \begin{tabular}{|l|l|}
$V_{3}$ & $V_{0}$ \\
\end{tabular} & \begin{tabular}{|l|l|}
10 & 10 \\
\end{tabular} & \begin{tabular}{|l|l|}
5 & 15 \\
\end{tabular} & \begin{tabular}{|l|l|}
15 & 5 \\
\end{tabular} & \begin{tabular}{|l|l|}
0 & 0 \\
\end{tabular} \\
\hline \begin{tabular}{|l|l|}
$V_{2}$ & $V_{1}$ \\
\end{tabular} & \begin{tabular}{|l|l|}
15 & 15 \\
\end{tabular} & \begin{tabular}{|l|l|}
10 & 20 \\
\end{tabular} & \begin{tabular}{|l|l|}
20 & 10 \\
\end{tabular} & \begin{tabular}{|l|l|}
25 & 25 \\
\end{tabular} \\
\hline \begin{tabular}{|l|l|}
$V_{3}$ & $V_{0}$ \\
\end{tabular} & \begin{tabular}{|l|l|}
15 & 15 \\
\end{tabular} & \begin{tabular}{|l|l|}
15 & 5 \\
\end{tabular} & \begin{tabular}{|l|l|}
20 & 10 \\
\end{tabular} & \begin{tabular}{|l|l|}
25 & 25 \\
\end{tabular} \\
\hline \begin{tabular}{|l|l|}
$V_{2}$ & $V_{1}$ \\
\end{tabular} & \begin{tabular}{|l|l|}
10 & 10 \\
\end{tabular} & \begin{tabular}{|l|l|}
20 & 10 \\
\end{tabular} & \begin{tabular}{|l|l|}
15 & 5 \\
\end{tabular} & \begin{tabular}{|l|l|}
0 & 0 \\
\end{tabular} \\
\hline$\#$ & 125 & 25 & 25 & 1 \\
\hline
\end{tabular}

(The first column of the table indicates that the intersection numbers are listed in accordance with Fig. 2.)

Proof. The description of the Hoffman-Singleton subgraphs in the proof of Theorem 5 leads immediately to all the entries of the table. Together with Lemma 32 it also justifies the claim about the two orbits. Addition of the entries for $V_{0}$ and $V_{1}$ does the rest.

\section{Actions of $A_{7}, P S L(4,2)$, and $A_{8}$}

Fig. 4 and its description in Section 8 show the alternating group $A_{7}$ acting simultaneously on sets of cardinalities $7,15,35$, and $42\left(S ; C_{1}\right.$ and $C_{2} ; L_{1}$ and $\left.L_{2} ; M\right)$. In this section we point out some well-known facts which manifest themselves in this view of the HigmanSims graph. For additional guidance, we include the distance distribution diagram around $w$ as Fig. 5.

We will indicate proofs of the various statements, to make clear how they all flow from this source. Alternative approaches to these themes can be found in $[9,11,20]$.

Lemma 43. The edges between $C_{2}$ and $L_{2}$ give the point-line incidence graph of the projective geometry $P G(3,2)$ with 15 points and 35 lines.

Proof. Since $H_{2}$ is an $\operatorname{srg}(50,7,0,1)$ and $C_{2}$ is a coclique in it, any two distinct vertices in $C_{2}$ have a unique common neighbour in $L_{2}$. To verify Veblen's axiom, one can proceed as follows. Note first that $v=(1,0,0) \in C_{1}$ has 8 neighbours in $C_{2}$ ( $v$ and $w$ are adjacent, hence their respective cocliques in $H_{2}$ meet in 8 vertices). It follows that $v$ has 7 neighbours in $L_{2}$; they are

$$
(2,0,0),(3,0,2),(3,0,-2),(3,1,2),(3,2,-2),(3,3,-2),(3,4,2) .
$$




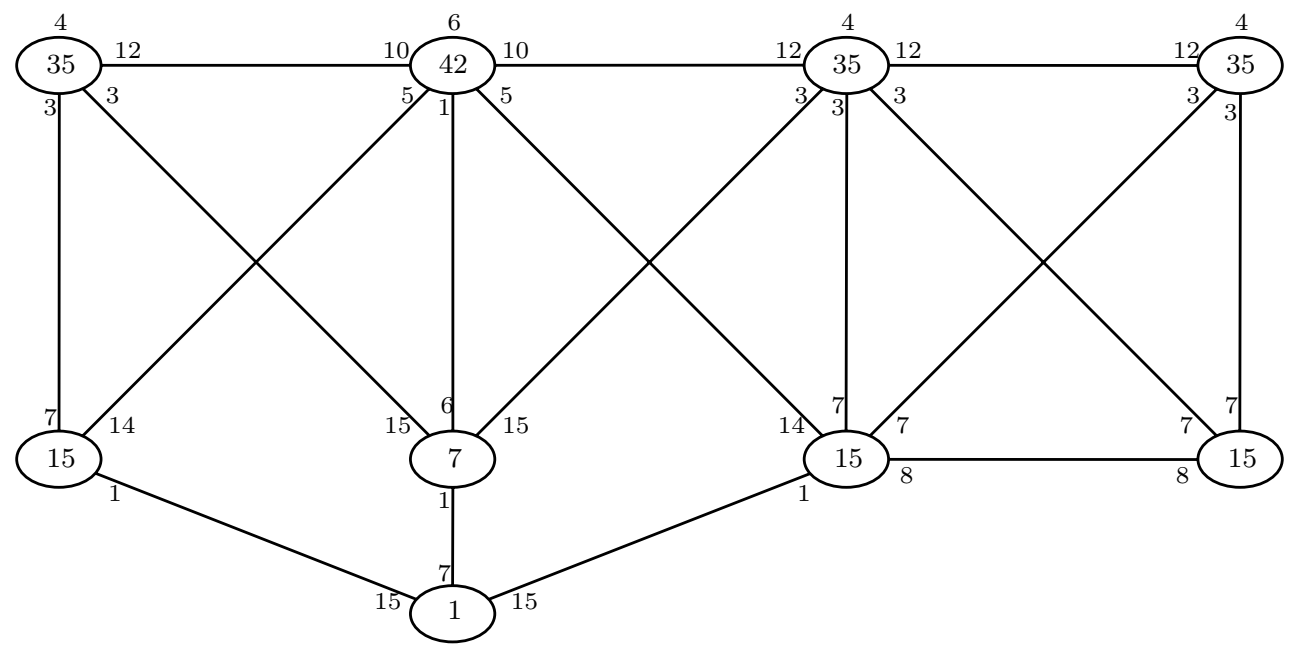

Figure 5: Distance distribution diagram in $K$

It turns out that these 7 vertices have just 7 neighbours in $C_{2}$, namely:

$$
(2,0,4),(2,3,0),(2,1,0),(2,4,0),(2,2,0),(2,0,1),(3,0,0) .
$$

Now it is easy to see that these 7 points and 7 lines form a Fano plane. As one lets $A_{7}$ act, one obtains the validity of Veblen's axiom in general.

Remark 44. In the preceding proof, each vertex of $C_{1}$ represents a Fano plane: its 7 neighbours in $L_{2}$ as lines, and vertices of $S$ as points. A further 15 such structures on $S$ are obtained starting with vertices of $C_{2}$. This demonstrates the well-known fact that there are 30 possible ways of defining a Fano plane on a 7-element set $(S)$, all equivalent under the action of the symmetric group $S_{7}$, but splitting into two orbits of length 15 under the action of $A_{7}$.

Remark 45. The construction of the Hoffman-Singleton graph from the 15 points and 35 lines of $\mathrm{PG}(3,2)$, where edges between points and lines indicate incidence, and edges between lines indicate disjointness of their corresponding triples, is also evident in the proof of Lemma 43. In addition, one sees again the bijection between lines of PG $(3,2)$ and triples of a 7-element set, in which intersecting lines correspond to triples with intersections of cardinality 1.

It is but a short step from here to establish the exceptional isomorphism of $\operatorname{PSL}(4,2)$ and $A_{8}$ : the automorphisms induced by $A_{7}$ are a subgroup of index 8 in the simple group $\operatorname{PSL}(4,2)$. The reader is encouraged to complete the story.

Remark 46. We note that the graph induced by $S \cup L_{2} \cup C_{2}$ is a trivial modification of Neumaier's Alt(7)-geometry ([38], see also [40], p.153, or [7], p.523; the usual convention is to have all edges $\{s, c\}$ for $s \in S, c \in C_{2}$; here, no such edges are present). 
Remark 47. In view of the presence of the group $A_{8}$ it is natural to ask if there is a way to construct the Alt(8)-geometry [38] (cf. also [40], p. 217) from the Higman-Sims graph $G$. The answer is indeed positive: one finds that the stabiliser in HS of an $\mathcal{F}_{2}$-coclique $C$ of $H_{1}$ is $A_{8}$, and that the HS-orbit of the set complement of $C$ in $H_{1}$ has length 8. The bijection between lines of PG $(3,2)$ and partitions of type $4^{2}$ of an 8 -element set becomes conspicuous. We will consider this in detail elsewhere.

\section{The Doubly Transitive Action of HS on 176 Points}

Since the Higman-Sims graph stood at the cradle of the sporadic simple group HS, a few words about the automorphism group are in order. Firstly, we note that as usual we can obtain the order of $\operatorname{Aut}(G)$ via the orbit-stabiliser theorem; we consider the action of $\operatorname{Aut}(G)$ on the Hoffman-Singleton subgraphs: $|\operatorname{Aut}(G)|=704 \cdot 126000=88704000$. The index 2 subgroup HS therefore has order 44352000 . The simplicity of HS is a consequence of the simplicity of the stabiliser (cf. Remark 49) of a Hoffman-Singleton subgraph when HS acts on one of its two orbits of Hoffman-Singleton subgraphs. We also note that whilst HS contains a subgroup which is isomorphic to the full automorphism group of the Hoffman-Singleton graph, there is no such subgroup of HS which acts on a Hoffman-Singleton subgraph of $G$.

G. Higman [24] discovered a doubly transitive permutation representation of the group HS (at the time it was still undecided whether the group he considered was in fact isomorphic to HS, though). We will show such an action in the framework of the Higman-Sims graph $G$.

Lemma 48. Let $S$ be the set of the 176 pairs of complementary Hoffman-Singleton subgraphs of $G$ in one of the two HS-orbits. Then the group HS acts doubly transitively on $S$.

Proof. We recall (cf. Lemma 42) that there are two orbits of Hoffman-Singleton graphs under the action of HS, and that the graphs occur in complementary pairs in each orbit. The two orbits are distinguished by the cardinalities of their intersection with a fixed Hoffman-Singleton subgraph of $G$. We also recall that the stabiliser of a HoffmanSingleton graph $H_{1}$ in HS is doubly transitive on the splits of $H_{1}$ into two $5 C_{5}$.

Looking at the table in (31), rows 1 and 2, we must show that any Hoffman-Singleton subgraph of $G$ with one of the intersection patterns in columns (a)-(c) can be transformed into any other by an automorphism of $G$ which stabilises $H_{1}$. This will be established if we can show that for any Hoffman-Singleton subgraphs $X, Y$ of $G$ such that $\mid X \cap$ $H_{1}|=| Y \cap H_{1} \mid=20$ there exists an automorphism $\tau$ in the stabiliser of $H_{1}$ such that $X^{\tau}=Y$. In other words: we need only consider row 1 , columns $(a)-(c)$. Remembering our automorphism $\psi$ from (4), we note that it suffices to prove transitivity of the stabiliser of $H_{1}$ on the patterns of columns $(a)$ and $(b)$.

Looking at column (a), note that the $15+10$ vertices of $X$ in $V_{3} \cup V_{0}$ are one half of a split of $V_{3} \cup V_{0}$ into a pair of $5 C_{5}$. This means that amongst the 10 vertices in $V_{0}$ there is 
a unique pair of adjacent ones, and affine transformations of $V_{0}$ induce a transitive action on the 125 sets of 10 vertices.

Considering column $(b)$, note that the 5 vertices of $X \cap V_{0}$ are part of an $\mathcal{F}_{2}$-coclique, and affine transformations with vertical eigenvalue 1 operate transitively on these.

Finally we must show that a pattern from $(a)$ can be transformed into one from column (b). To this end we return to the example in Remark 4. The automorphism (of $H_{1}$; but note that it can be extended to $G$ ) defined by (6) will turn the pattern of 20 boldface positions into a pattern of 5 vertices in $V_{0}$ and 15 vertices of $V_{1}$. It remains to show that the 20 boldface positions are the intersection of a Hoffman-Singleton subgraph with $H_{1}$. To this end we define an automorphism $\tau$ of $G$ which preserves $V_{3} \cup V_{0}$ and such that $V_{3}^{\tau}$ and $V_{2}^{\tau}$ each have 10 vertices in $V_{0}$, resp. $V_{1}$. This can be achieved following the method of Remark 4: choose the Petersen graph consisting of the vertices $(3,0,0)-(3,0,4)$ and $(0,0,0)-(0,0,4)$ and define its image so that two $V_{3}$-vertices of $P$ are mapped onto the bold positions. If we also require that $(0,1,0)$ maps to $(0,2,1)$, we obtain the following automorphism $\tau$ :

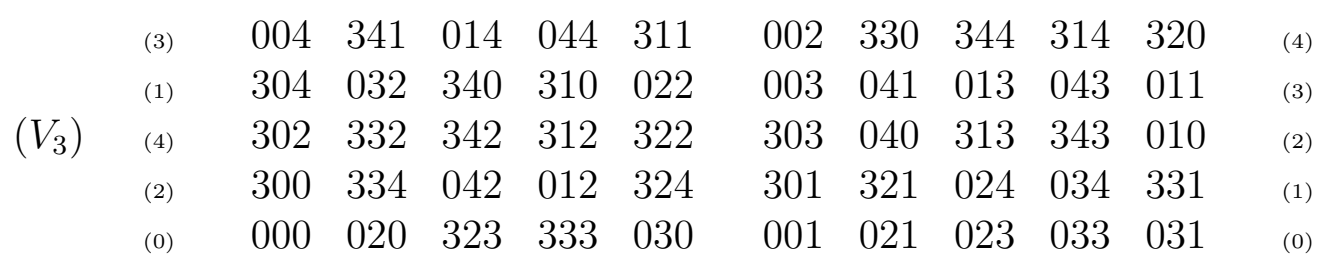

It is easy to verify that $V_{3}^{\tau}$ has 10 vertices in $V_{0}$, and that $\tau$ can be extended to an automorphism of $G$.

Remark 49. In [16] we showed how to use Definition 1 to obtain that the order of the automorphism group of the Hoffman-Singleton graph is 252000 . We now show that this automorphism group has a subgroup of index 2 which is simple. (This is of course a well-known fact, usually by reference to [22], but we want to show that all the arguments can proceed at a very elementary level.)

The automorphism of the Hoffman-Singleton graph $H$ induced by $(0, x, y) \mapsto(0, x,-y)$ interchanges the two families of maximum cocliques. Therefore those automorphisms which preserve the two families of maximum cocliques form a subgroup $U$ of index 2 in $\operatorname{Aut}(H)$, which is therefore of order 126000 . Since the transposition $(0, x, y) \mapsto(0, x,-y)$ does not belong to the vertex stabiliser of $(0,0,0)$ in this subgroup, this stabiliser must have index 2 in the vertex stabiliser of the full automorphism group, which is the symmetric group $S_{7}$. Since $U$ is primitive on the vertices of $H$, and $A_{7}$ is simple, we conclude that $U$ is a simple group of order 126000 . In order to identify the isomorphism type of this simple group $(P S U(3,5))$, we refer to O'Nan [39] or D.G. Higman [22].

\section{Coordinate-free Description of $G$}

We have noted that the edges between $V_{0}$ and $V_{1}$ describe the incidence graph of a biaffine plane of order $5, V_{0}$ being the set of points, $V_{1}$ the set of lines. The edges between $V_{0}$ 
and $V_{3}$ form the incidence graph of points and the set of parabolas $y=(x-a)^{2}+b$ in this biaffine plane. Further, the edges between $V_{1}$ and $V_{2}$ describe the incidence graph of 'dual points' and a certain set of 'dual parabolas' of the biaffine plane. (Dual points are, as usual, the lines of the geometry, and dual parabolas are sets of all tangents of a parabola - it is easy to show that the set of tangents to the parabola $y=3(x-a)^{2}+b$ consists of all lines $y=m x+c$ where $c=2(m+a)^{2}+b-2 a^{2}$.)

The following theorem provides geometric descriptions for all adjacencies between $V_{i}$ and $V_{j}(i \neq j)$.

Theorem 6. Interpret the sets $V_{0}, \ldots, V_{3}$ as above, with $p, \ell, P, Q$ be elements of $V_{0}, \ldots, V_{3}$ respectively. Then

1. the parabola $P$ is adjacent (in $G$ ) to the dual parabola $Q$ if and only if exactly one of the lines of $Q$ is a tangent of $P$;

2. the point $p$ is adjacent in $G$ to the dual parabola $Q$ if and only if $p$ does not lie on any of the lines of $Q$ (i.e. $p$ is an internal point of the dual parabola $Q$ );

3. the line $\ell$ is adjacent in $G$ to the parabola $P$ if and only if $\ell$ is a passant of $P$ (cf. end of Section 5);

4. all other adjacencies in $G$, apart from the 5-cycles within each of $V_{0}, \ldots, V_{3}$, describe incidences.

Proof. It suffices to indicate a proof of the first statement. Statements 2 and 3 are duals of each other, and statement 3 was established at the end of Section 5 . To establish statement 1 , assume that $P$ has the equation $y=(x-a)^{2}+b$ and $Q$ is given by $c=2(m-A)^{2}+B$. Then $Q$ consists of the lines $y=m x+2(m-A)^{2}+B$. Such a line is a tangent of $P$ if it has a unique point of intersection with $P$; i.e. the equation $(x-a)^{2}+b=m x+2(m-A)^{2}+B$ has discriminant 0 (as equation in $x$ ). This leads to the condition

$$
m^{2}+(a+A) m-b+2 A^{2}+B=0 .
$$

This quadratic equation in $m$ has a unique solution if and only if its discriminant equals 0 :

$$
(a+A)^{2}-b+2 A^{2}+B=0 .
$$

This is the condition of adjacency between vertices $(3, a, b)$ and $(2, A, B)$.

Remark 50. This description opens the way to define families of generalised HigmanSims graphs, starting from any McKay-Miller-Širáň graph instead of $H$ (or more generally, starting from any graph based on the incidence graph of a biaffine plane).

Remark 51. As a point of interest we note that the sets $V_{0}, \ldots, V_{3}$ (as geometric entities in a biaffine plane) have been considered by Wild $[49,50]$. The incidence graphs of the systems $S\left(C_{1}, C_{2}\right)$ of Wild are obtained by removing all edges within each of $V_{0}, \ldots, V_{3}$, and removing the (diagonal) edges between $V_{0}$ and $V_{2}$, and between $V_{1}$ and $V_{3}$. 
Wild [50] also establishes the isomorphism of the graphs induced by $V_{0} \cup V_{1}$ and $V_{0} \cup V_{3}$ (points and lines vs points and conics, omitting the 5-cycles; the generalisation from $q=5$ to general $q$ is obvious). This is the biaffine analogue of results for projective planes [33, 41, 42]. See also [43] for recent work.

Remark 52. Further to Remark 51, we note that the isomorphism between the graphs induced by $V_{0} \cup V_{1}$ and $V_{0} \cup V_{3}$ manifested itself in [48] (cf. also [17]), disguised by the language of voltage assignments. In this paper, Šiagiová expressed certain adjacencies of the McKay-Miller-Širán graphs [36, 19] by means of quadratic equations, whereas the original definition uses linear equations which are the direct analogue of the equations $y=m x+c$ in Robertson's definition of the Hoffman-Singleton graph. One might say that the original description operates in $V_{0} \cup V_{1}$, whilst Šiagiová's description uses $V_{0} \cup V_{3}$. (Of course, the present paper considers only the case $q=5$; [48] deals with arbitrary $q \equiv 1$ $\bmod 4$.)

\section{Decomposition of the Higman-Sims Graph into Five Isomorphic Subgraphs}

In the course of our studies of the Higman-Sims graph, the following decomposition into 5 isomorphic subgraphs of order 20 appeared.

Lemma 53. Let

$$
W_{i}=\bigcup_{r \in \mathbb{Z}_{5}}\{(0, i, r),(1,3 i, r),(2,2 i, r),(3,2 i, r)\}, \quad\left(i \in \mathbb{Z}_{5}\right) .
$$

For $i \in \mathbb{Z}_{5}$ the subgraphs of $G$ induced by $W_{i}$ are isomorphic to the Kronecker product of the Petersen graph with a coclique of order 2.

Proof. Each $W_{i}$ consists of four 5 -cycles, one from each $V_{k}, k=1, \ldots, 4$. For each pair of (cyclically) consecutive values of $k$ the two 5-cycles form a Petersen graph. One sees without difficulty that $(0, i, r)$ is adjacent to $(2,2 i, s)$ if and only if $r=s \pm 1$, i.e. if and only if $(0, i, r)$ is adjacent to $(0, i, s)$; and $(0, i, r)$ is adjacent to $(3,2 i, s)$ if and only if $r=s+i^{2}$, i.e. if and only if $(0, i, r)$ is adjacent to $\left(1,3 i, r+2 i^{2}\right)$. The remaining details can be left to the reader.

Remark 54. The subgraph of order 80 induced by four of these subgraphs $W_{i}$ is identified as the second orbit of the stabiliser of the remaining one of these graphs in [4]. What is new here is the observation that we are actually dealing with five isomorphic subgraphs of order 20 .

As an aside, we add that the orbit of $W_{0}$ under $\operatorname{Aut}(G)$ as well as under HS has length 5775 , the number of elements in one of the two conjugacy classes of involutions in HS. Indeed, each such involution has one of these 20-vertex subgraphs as fixed-point set. For example, $W_{0}$ is the fixed-point set of the automorphism $\psi^{4}$ as mentioned in Remark 11. (The second class of involutions of HS can be represented by $h^{2}$ (cf. Lemma 12) which is fixed-point-free.) 


\section{Historical Comment}

T.B. Jajcayová and R. Jajcay, in their recent biographical note [29] on D.M. Mesner, report that the Higman-Sims graph made an early appearance in Mesner's 1956 dissertation, getting a brief mention as $N L_{2}(10)$ in [37] (designs of negative Latin square type). However, the automorphism group of the graph was not considered in either work. We add that in [47], p.107, the Higman-Sims graph is indeed identified as $N L_{2}(10)$.

An interesting first-hand account (in English) by C.C. Sims on how the Higman-Sims group was found is included in a forthcoming paper by G. Hiss [25].

\section{Acknowledgements}

R.H. Jeurissen's report [31] was a source of inspiration for this work. After the bulk of this paper was completed, I also became aware of A.E. Brouwer's suite of web pages [4] which are a mine of valuable information, presenting some of these results in a different light. The computer algebra system MAGMA [3] was used for the exploration of the graphs.

I wish to thank Cheryl Praeger for her interest in this work, and in particular for communicating her result in Theorem 3. Thanks also to M.A. Fiol who supplied me with a copy of [31]. Many thanks to Alice Karetai who worked with me in the framework of an undergraduate summer studentship, proving Theorem 1, counting cycles, and more. In particular she derived the equations for adjacency in $H_{3}$. I am grateful to my Department for this support.

Last but by no means least, I am grateful to an anonymous referee whose thorough reading of this paper lead to a number of improvements.

\section{References}

[1] T. Beth, D. Jungnickel, and H. Lenz. Design theory. Vol. I. Cambridge University Press, Cambridge, second edition, 1999.

[2] N.L. Biggs and A.T. White. Permutation groups and combinatorial structures. Cambridge University Press, Cambridge, 1979.

[3] W. Bosma and J.J. Cannon. Handbook of Magma Functions. University of Sydney, 1996.

[4] A. E. Brouwer. A slowly growing collection of graph descriptions. http://www.win.tue.nl/ ${ }^{\sim}$ aeb/drg/graphs/.

[5] A. E. Brouwer and J. H. van Lint. Strongly regular graphs and partial geometries. In Enumeration and design (Waterloo, Ont., 1982), pages 85-122. Academic Press, Toronto, ON, 1984. 
[6] A.E. Brouwer, A.M. Cohen, and A. Neumaier. Distance-regular graphs. SpringerVerlag, Berlin, 1989.

[7] F. Buekenhout, editor. Handbook of incidence geometry. North-Holland, Amsterdam, 1995. Buildings and foundations.

[8] P.J. Cameron and J.H. van Lint. Designs, graphs, codes and their links. Cambridge University Press, Cambridge, 1991.

[9] G. M. Conwell. The 3-space PG(3, 2) and its group. Annals of Math. (2), 11:60-76, 1910.

[10] R.T. Curtis. Symmetric generation of the Higman-Sims group. J. Algebra, 171(2):567-586, 1995.

[11] W. L. Edge. The geometry of the linear fractional group LF(4,2). Proc. London Math. Soc. (3), 4:317-342, 1954.

[12] A. Gewirtz. Graphs with maximal even girth. Canad. J. Math., 21:915-934, 1969.

[13] C. Godsil and G. Royle. Algebraic graph theory. Springer-Verlag, New York, 2001.

[14] W.H. Haemers. Eigenvalue techniques in design and graph theory. Mathematisch Centrum, Amsterdam, 1980.

[15] P.R. Hafner. Files and links related to the Higman-Sims graph.

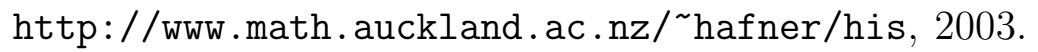

[16] P.R. Hafner. The Hoffman-Singleton graph and its automorphisms. J. Algebraic Combin., 18(1):7-12, 2003.

[17] P.R. Hafner. On the graphs of McKay-Miller-Širáň. In Sirocco 8 (F. Comellas, J. Fàbrega, P. Fraignaud eds.), pages 209-215, Carleton Scientific, 2001.

[18] P.R. Hafner. From the Steiner system $S(2,5,21)$ to $S(5,8,24)$ via the Higman-Sims graph. In preparation.

[19] P.R. Hafner. Geometric realisation of the graphs of McKay-Miller-Širáň. J. Combin. Theory Ser. B, 90(1):223-232, 2004.

[20] J.I. Hall. On identifying PG(3, 2) and the complete 3-design on seven points. Ann. Discrete Math., 7:131-141, 1980. Topics on Steiner systems (C.C.Lindner, A.Rosa eds.).

[21] A. Heinze. Applications of Schur rings in algebraic combinatorics : graphs, partial difference sets and cyclotomic schemes. 189pp., Dissertation, Oldenburg, 2001. http://docserver.bis. uni-oldenburg.de/publikationen/dissertation/ fb06.html. 
[22] D.G. Higman. Finite permutation groups of rank 3. Math. Z., 86:145-156, 1964.

[23] D.G. Higman and C.C. Sims. A simple group of order 44,352,000. Math. Z., 105:110-113, 1968.

[24] G. Higman. On the simple group of D. G. Higman and C. C. Sims. Illinois J. Math., 13:74-80, 1969.

[25] G. Hiss. Die sporadischen Gruppen. Jahresber. Deutsch. Math.-Verein., 105:169193, 2003) http://www.math.rwth-aachen.de/ ${ }^{\sim}$ Gerhard.Hiss/Preprints/giessen.ps.

[26] A.J. Hoffman and R.R. Singleton. On Moore graphs with diameters 2 and 3. IBM J. Res. Develop., 4:497-504, 1960.

[27] D.R. Hughes. Semisymmetric 3-designs. In Finite geometries (Pullman, Wash., 1981), pages 223-235. Dekker, New York, 1983.

[28] D.R. Hughes and F.C. Piper. Design theory. Cambridge University Press, Cambridge, 1985.

[29] T.B. Jajcayová and R. Jajcay. On the contributions of Dale Marsh Mesner. Bull. Inst. Combin. Appl., 36:46-52, 2002.

[30] L.O. James. A combinatorial proof that the Moore $(7,2)$ graph is unique. Utilitas Math., 5:79-84, 1974.

[31] R.H. Jeurissen. Topography of the Hoffman-Singleton graph. Technical Report 8324, Department of Mathematics, Catholic University, Nijmegen, 1983.

[32] L.K. Jørgensen and M. Klin. Switching of edges in strongly regular graphs. I. A family of partial difference sets on 100 vertices. Electron. J. Combin., 10(1):Research Paper 17, 31 pp. (electronic), 2003.

[33] D. Jungnickel and K. Vedder. On the geometry of planar difference sets. European J. Combin., 5(2):143-148, 1984.

[34] K.N. Majindar. On the parameters and intersection of blocks of balanced incomplete block designs. Ann. Math. Statist., 33:1200-1205, 1962.

[35] R. Mathon and A.P. Street. Partitions of sets of two-fold triple systems, and their relation to some strongly regular graphs. Graphs Combin., 11(4):347-366, 1995.

[36] B.D. McKay, M. Miller, and J. Širáň. A note on large graphs of diameter two and given maximum degree. J. Combin. Theory Ser. B, 74(1):110-118, 1998.

[37] D.M. Mesner. A new family of partially balanced incomplete block designs with some Latin square design properties. Ann. Math. Statist, 38:571-581, 1967. 
[38] A. Neumaier. Some sporadic geometries related to PG(3, 2). Arch. Math. (Basel), 42(1):89-96, 1984.

[39] M.E. O'Nan. Automorphisms of unitary block designs. J. Algebra, 20:495-511, 1972.

[40] A. Pasini. Diagram geometries. The Clarendon Press Oxford University Press, New York, 1994.

[41] G. Pickert. Differenzmengen und Ovale. In Proceedings of the Oberwolfach Meeting "Kombinatorik" (1986), volume 73, pages 165-179, 1989.

[42] G. Pickert. Doppelebenen und Loops. J. Geom., 41(1-2):133-144, 1991.

[43] B. Polster and A.E. Schroth. Split semi-biplanes in antiregular generalized quadrangles. Bull. Belg. Math. Soc. Simon Stevin, 4(5):625-637, 1997.

[44] C.E. Praeger and C. Schneider. Private communication. 13 Nov 2002.

[45] N. Robertson. Graphs Minimal Under Girth, Valency, and Connectivity Constraints. Phd thesis, University of Waterloo, 1969.

[46] B. Segre. Ovals in a finite projective plane. Canad. J. Math., 7:414-416, 1955.

[47] M.S. Shrikhande and S.S. Sane. Quasi-symmetric designs. Cambridge University Press, Cambridge, 1991.

[48] J. Šiagiová. A note on the McKay-Miller-Širáň graphs. J. Combin. Theory Ser. B, 81(2):205-208, 2001.

[49] P. Wild. Divisible semibiplanes and conics of Desarguesian biaffine planes. Simon Stevin, 58(1-2):153-166, 1984.

[50] P. Wild. Biaffine planes and divisible semiplanes. J. Geom., 25(2):121-130, 1985.

[51] R.A. Wilson, Vector stabilizers and subgroups of Leech lattice groups. J. Algebra, 127(2):387-408, 1989.

[52] S. Yoshiara. A locally polar geometry associated with the group HS. European J. Combin., 11(1):81-93, 1990. 